\title{
Ion and neutral molecules in the W43-MM1(G30.79 FIR 10) infalling clump
}

\author{
Paulo C. Cortes ${ }^{1}$ \\ National Radio Astronomy Observatory - Joint ALMA Office, Alonso de Cordova 3107, \\ Vitacura, Santiago, Chile \\ pcortes@alma.cl
}

Received __ ; accepted _ 


\begin{abstract}
The high mass star forming clump W43-MM1 has been mapped in $\mathrm{N}_{2} \mathrm{H}^{+}(4 \rightarrow 3)$, $\mathrm{C}^{18} \mathrm{O}(3 \rightarrow 2), \mathrm{SiO}(8 \rightarrow 7)$, and in a single pointing in $\mathrm{DCO}^{+}(5 \rightarrow 4)$ towards the center of the clump. Column densities from these observations as well as previous $\mathrm{HCO}^{+}(4 \rightarrow 3), \mathrm{H}^{13} \mathrm{CO}^{+}(4 \rightarrow 3), \mathrm{HCN}(4 \rightarrow 3), \mathrm{H}^{13} \mathrm{CN}(4 \rightarrow 3)$, and $\mathrm{CS}(7 \rightarrow 6)$ data, have been derived using the RADEX code, results later used to derived chemical abundances at selected points in the MM1 main axis. Comparing with chemical models, we estimate an evolutionary age of $10^{4}$ years for a remarkable warm hot core inside MM1. We also proposed that the dust temperature derived from SED fitting in MM1 is not representative of the gas temperature deep inside the clump as dust emission may have become optically thick. By deriving a deuterium fractionation of $1.2 \times 10^{3}$, we estimate an electron fraction of $X(e)=6.5 \times 10^{-8}$. Thus, the coupling between the neutral gas and the magnetic field is estimated by computing the ambipolar diffusion Reynolds number $R_{m}=18$ and the wave coupling number $W=110$. Considering that the infalling speed is slightly supersonic $(M=1.1)$ but sub-alfvenic, we conclude that the MM1 clump has recently or is in the process of decoupling the field from the neutral fluid. Thus, the MM1 clump appears to be in an intermediate stage of evolution in which a hot core has developed while the envelope is still infalling and not fully decoupled from the ambient magnetic field.
\end{abstract}

Subject headings: ISM: Abundances, ISM: Magnetic Fields, ISM: clouds, ISM: Kinematics and dynamics 


\section{Introduction}

High mass star formation involves the study of gas masses many orders of magnitude larger than low mass stars increasing the complexity as the process is highly energetic and dynamic. The distances at which the regions of interest are located are usually kilo-parsecs away from the sun, complicating the use of single dish telescopes as current dish sizes do not produce enough angular resolutions to resolve individual cores. Thus, the emission is often diluted within the beam complicating the interpretation as the high mass cores inside clumps are usually shrouded by optically thick envelopes, even with dust emission. We know that massive stars $\left(\gtrsim 10 \mathrm{M}_{\odot}\right)$ form in giant molecular clouds several orders of magnitude larger than the low-mass star forming clouds. As it is still not clear how these giant ensembles of gas came to be, we know that their location are correlated with the spiral arms of our galaxy. These massive complexes are known to harbor cores with usually several sources as massive stars are born in groups or associations. Once a massive star turns on it will quickly perturb and ionize the surrounding envelope creating what it is known as a hot core (Wood \& Churchwell 1989; Hoare et al. 2007). The intense radiation coming from these young sources, will evaporate $\mathrm{C}$ and $\mathrm{O}$ bearing molecules from ice mantles surrounding dust grains, changing dramatically the chemical diversity around the star(s) (Herbst \& van Dishoeck 2009). Even though, the inventory of complex molecules in hot cores have been growing consistently in the past 10 years, still chemical and dynamical models have problems trying to reproduce abundances and explain the chemical differentiation seen in high mass cores (Garrod et al. 2008). Thus, probing the densest regions becomes paramount to understand their physical and chemical conditions. On the otherhand, the complete set of physical parameters have to be considered if we want to fully understand the process. Magnetic fields are known to be ubiquitous in the ISM and giant molecular clouds are no exception. Strong magnetic field strengths have been measured from CN Zeeman splitting towards high mass star forming regions to be in the order of milligauss (Crutcher et al. 1996, 1999). Also, observations done from polarized dust emission find ordered field structure with strength estimations around the 
same values (Lai 2001; Cortes et al. 2005; Cortes \& Crutcher 2006; Cortes et al. 2008). Thus, incorporating the magnetic field along with the set of parameters used to study the star formation process is critical.

In this paper we present new observations of $\mathrm{DCO}^{+}(5 \rightarrow 4), \mathrm{N}_{2} \mathrm{H}^{+}(4 \rightarrow 3)$, and $\mathrm{SiO}(8 \rightarrow 7)$ as well as re-processed $1 \mathrm{HCO}^{+}(4 \rightarrow 3), \mathrm{H}^{13} \mathrm{CO}^{+}(4 \rightarrow 3), \mathrm{HCN}(4 \rightarrow 3), \mathrm{H}^{13} \mathrm{CN}(4 \rightarrow 3)$, and $\mathrm{CS}(7 \rightarrow 6)$ observations done previously by Cortes et al. (2010) and hereafter PCI, from the high mass star forming clump W43-MM1 (also known as G30.79 FIR 10). The W43-MM1 is a large high mass star forming clump located within the W43 region near $l=31^{\circ}, b=0^{\circ}$. Liszt (1995) observed the W43 molecular complex in $\mathrm{HCO}^{+}$and ${ }^{13} \mathrm{CO}$ finding a series of several rings and shells in the dense molecular gas, which they attributed to be a product of star formation. Mooney et al. (1995) made the first dust continuum observations of this source at $1.3 \mathrm{~mm}$ using the IRAM 30-meter telescope. They detected a total flux of 13.6 Jy positioning MM1 at an interface with the extended $\mathrm{H}$ II region in the W43 complex. Several $\mathrm{H}_{2} \mathrm{O}$ masers (Cesaroni et al. 1988) have also been detected within an arcsecond from the dust peak found in the $1.3 \mathrm{~mm}$ data. No centimeter radio-continuum emission seems to be associated with MM1, which suggests that the source is in an early stage of evolution. Additional continuum mapping has been done by Motte et al. (2003) at $1.3 \mathrm{~mm}$ and $350 \mu \mathrm{m}$ with the IRAM 30-m and CSO telescopes 2 respectively. They determined a $\mathrm{v}_{l s r}=98.8 \mathrm{~km} \mathrm{~s}^{-1}$ from $\mathrm{H}^{13} \mathrm{CO}^{+}$and a dust temperature of $\mathrm{T}_{\text {dust }} \sim 19 \mathrm{~K}$, by fitting a grey body model to the spectral energy density distribution. Cortes \& Crutcher (2006) made interferometric observations of polarized dust emission with BIMA, finding an ordered pattern for the field and estimating and deriving a total mass of about $3000 \mathrm{M}_{\odot}$ for the MM1 clump.

\footnotetext{
${ }^{1}$ By re-processing, we mean using the data cubes obtained by PCI to produce maps and calculate column densities with RADEX (see section 2 which have not been done previously

${ }^{2}$ IRAM stands for Institut de Radioastronomie Millimetrique, and CSO stands for Caltech Submillimeter Observatory
} 
Finally, PCI studied the gravitational equilibrium of this source finding compelling evidence for infalling motions and refining a previous estimation of the magnetic field strength in the plane of the sky (Cortes \& Crutcher 2006) to $B=855 \mu \mathrm{G}$. PCI also produced several maps from the previously mentioned molecular species. From this set of observations, we have derived chemical abundances as well as spatial distributions of molecules, which we use to estimate the electron fraction and the coupling parameters between the magnetic and the neutral gas. The paper is organized as follows, Sect. 1 is the introduction, Sect. 2 presents the observation configuration, Sect. 3 the observational results, while in Sect. 4 we discuss the chemical abundances, the spatial differentiation, the fractional ionization, and the ion-neutral coupling. Finally, Sect. 5 presents the summary and conclusions.

\section{Observation Configuration}

\subsection{ASTE Observations}

The W43-MM1 clump was observed during July 2010 using the Atacama Sub-millimeter Telescope Experiment (ASTE) from the National Astronomical Observatory of Japan (NAOJ) (Kohno 2005). The telescope is located at Pampa la bola in the Chilean Andes plateau reserve for Astronomical research at 4900 meters of altitude. ASTE is a $10 \mathrm{~m}$ diameter antenna equipped with a $345 \mathrm{GHz}$ double side band SIS-mixer receiver. We observed $\mathrm{DCO}^{+}(5 \rightarrow 4)$ and $\mathrm{N}_{2} \mathrm{H}^{+}(4 \rightarrow 3)$ by tuning the CAT345 receiver to the appropriate frequencies (see Table 1 for frequencies and map sizes) giving a beam size of $\sim 20^{\prime \prime}$, and a velocity resolution of $0.1 \mathrm{~km} \mathrm{~s}^{-1}$. The MAC (a XF-type digital spectro-correlator) was set to high resolution mode with a total bandwidth of $128 \mathrm{MHz}$. The pointing accuracy was in the order of $2^{\prime \prime}$, with VY-CMa used as the pointing source. The observations were done by performing single pointing (position switching) with an off position carefully selected to avoid contamination to the on position. Also, a small $60^{\prime \prime} \times 60^{\prime \prime}$ map for $\mathrm{N}_{2} \mathrm{H}^{+}$was obtained centered at the reference position. The observations 
were done under excellent weather conditions (precipitable water vapor or $\langle\mathrm{PWV}\rangle \quad 0.5 \mathrm{~mm}$ and wind speed under $3 \mathrm{~m} / \mathrm{s})$. Our reference position was $(\alpha, \delta)=\left(18^{\mathrm{h}} 47^{\mathrm{m}} 46.9^{\mathrm{s}},-1^{\circ} 54^{\prime} 29 \cdot 1^{\prime \prime}\right)$ (J2000), which coincides with the peak dust emission reported and used by Mooney et al. (1995); Motte et al. (2003); Cortes \& Crutcher (2006); Cortes et al. (2010); while the OFF position used is $(\alpha, \delta)=\left(18^{\mathrm{h}} 49^{\mathrm{m}} 46.7^{\mathrm{s}},-2^{\circ} 00^{\prime} 52.0^{\prime \prime}\right)$ Initial data reduction and calibration was done using the NEWSTAR package, and the calibrated data were later exported into our own software for analysis and plotting.

\subsection{APEX observations}

Observations were performed during the first week of August 2008 using the Swedish Heterodyne Facility Instrument (SHFI) mounted on the Atacama Pathfinder Experiment telescope (APEX) (Güsten et al. 2006), located at llano de Chajnantor also in the Chilean Andes plateau reserve for Astronomical research at. The SHFI was tuned to $347.33 \mathrm{GHz}$ to detect the $\mathrm{SiO}(J=8 \rightarrow 7)$ molecular transition. The spectrometer was set up to 8192 channels with a resolution of $0.1 \mathrm{~km} \mathrm{~s}^{-1}$. beam efficiency is $\eta=0.73 \pm 0.07$ as measured by the APEX staff, with a pointing accuracy better than $2^{\prime \prime}$ and a beam size of $19^{\prime \prime}$. The APEX wobbler was not used for this observations. Jupiter and R-Aql were used as intensity and pointing calibrators where the observations were calibrated by the usual chopper-wheel method. The observations were done in raster mode with spacings of $15^{\prime \prime}$ from the same reference position used for the ASTE observations. The initial data reduction was done with the GILDAS-CLASS reduction package and the final analysis with our own software tools. 
Table 1. Observing parameters for molecular lines observations towards W43-MM1.

\begin{tabular}{cccccccccc}
\hline \hline Line & Transition & $\begin{array}{c}\text { Frequency } \\
{[\mathrm{GHz}]}\end{array}$ & $\begin{array}{c}\text { Beam Size } \\
{[\mathrm{arcsec}]}\end{array}$ & Area mapped & $\begin{array}{c}\text { Peak } \\
{[\mathrm{K}]}\end{array}$ & $\begin{array}{c}\text { Line-width } \\
{\left[\mathrm{km} \mathrm{s}^{-1}\right]}\end{array}$ & $\begin{array}{c}\int \mathrm{T} d v \\
{[\mathrm{Kkm} / \mathrm{s}]}\end{array}$ & $\begin{array}{c}\sigma \\
{[\mathrm{K}]}\end{array}$ & Telescope \\
\hline & & & & & & & & & \\
$\mathrm{DCO}^{+}$ & $(J=5 \rightarrow 4)$ & 360.1698810 & 20.9 & single pointing & 0.044 & 2.9 & 0.11 & 0.01 & ASTE \\
$\mathrm{N}_{2} \mathrm{H}^{+}$ & $(J=4 \rightarrow 3)$ & 372.6725090 & 20.3 & $60^{\prime \prime} \times 60^{\prime \prime}$ & 2.1 & 3.7 & 7.3 & 0.08 & ASTE \\
$\mathrm{SiO}$ & $(J=8 \rightarrow 7)$ & 347.3305786 & 21.7 & $60^{\prime \prime} \times 60^{\prime \prime}$ & 0.6 & $\mathrm{n} / \mathrm{a}$ & $\mathrm{n} / \mathrm{a}$ & 0.24 & APEX \\
\hline
\end{tabular}




\section{Results}

\subsection{Neutrals Observations}

In PCI, we reported a single $30^{\prime \prime} \times 30^{\prime \prime}$ averaged spectrum, centered at the MM1 reference position, for $\mathrm{C}^{18} \mathrm{O}(3 \rightarrow 2)$. Even though we mapped a larger area of $150^{\prime \prime} \times 120^{\prime \prime}$ sampled every $15^{\prime \prime}$, we did not present the complete set of observations in that work. Here, we present the integrated intensity map in Figure 1. The map follows the elongated structure seen from the ${ }^{12} \mathrm{CO}(3 \rightarrow 2)$ map presented in PCI (see Figure 2 there). Two clear peaks are seen in the $\mathrm{C}^{18} \mathrm{O}$ map, one is coincident with the reference position and the other is located at $(\alpha, \delta)=\left(-120^{\prime \prime},-45^{\prime \prime}\right)$. The first peak agrees with the peak in the dust emission, $\mathrm{HCN}(4 \rightarrow 3), \mathrm{HCO}^{+}(4 \rightarrow 3)$, and $\mathrm{CS}(7 \rightarrow 6)$ presented in PCI. The second and strongest peak is interesting as it is located at the edge of the map following the ${ }^{12} \mathrm{CO}(3 \rightarrow 2)$ peak where it appears to be anti-correlated with the dust emission i.e. the dust emission is minimum where $\mathrm{CO}$ is maximum. According to the continuum maps of MM1 done by Motte et al. (2003) $1.3 \mathrm{~mm}$ and $350 \mu \mathrm{m}$, the dust emission decreases an order of magnitude with respect to the dust-peak at this position. As previously suggested by PCI, this area seems to be at an interface with the H II region produced by a large cluster of massive stars located at the center of W43 (Wilson et al. 1970; Smith et al. 1978). Thus, it it possible that we are seeing part of a PDR at that area.

We observed an area of $60^{\prime \prime} \times 60^{\prime \prime}$ in $\mathrm{SiO}(8 \rightarrow 7)$ towards the center of the MM1 clump (see Figure 2). Large amount of data were discarded as the baselines showed evident ripples which we could not remove. There is no conclusive evidence of $\mathrm{SiO}$ emission from these data, only an upper limit of $0.6 \mathrm{~K}$ around the systemic velocity of MM1 is obtained with an rms noise of $0.24 \mathrm{~K}$ which is less than $3 \sigma$. Thus, we are not able to confirm the marginal evidence find by PCI from the $\mathrm{HCO}^{+}$and $\mathrm{CS}$ line-wings. However, new un-published $\mathrm{SMA} 3$ interferometric observations

\footnotetext{
${ }^{3}$ SMA stands for Sub Millimeter Array
} 
(Sridharan to be submitted 2011) found evidence for outflow emission towards MM1 as the interferometer filters the envelope probing angular scales closer to the center of the core. Thus, it is likely that the outflow is young and deeply embedded into the clump with its emission diluted within the APEX beam.

\subsection{Ion observations}

Single pointing observations were obtained towards the W43-MM1 core in order to detect the $\mathrm{DCO}^{+}(5 \rightarrow 4)$ molecular transition. Figure 3 shows the spectrum obtained and its corresponding Gaussian fit (in red); Table 1 summarizes the main molecular line parameters. With a single peak of $44 \mathrm{mK}$ over a noise level of $\sigma=10 \mathrm{mK}$, the line intensity corresponds to a $4 \sigma$ significance observation, which is clearly a detection. The data were processed by keeping only spectra with stable baselines using order 1 polynomial fits. The spectrum was binned every 10 channels, giving a velocity resolution of $1 \mathrm{~km} \mathrm{~s}^{-1}$. The $\mathrm{DCO}^{+}$molecule has been detected in its lower transition towards a number of star forming cores with similar results to our own (Wootten et al. 1979; Caselli et al. 2002a.b). The line profile is clearly Gaussian, which suggests that the line is likely optically thin. Even though it is expected to find high abundances of $\mathrm{DCO}^{+}$in early stages of massive cores, the abundances are unlikely to be large enough to make the line optically thick. By looking at Figure 1 we also noticed a strong line besides the $\operatorname{DCO}^{+}(5-4)$ detection (with a Gaussian fit in blue). The corresponding line center frequency from the best fit is $360.1847 \mathrm{GHz}$, it is currently un-identified and its nature will be discussed in section 4.1 .

We observed $\mathrm{N}_{2} \mathrm{H}^{+}(4 \rightarrow 3)$ rotational transition towards the center of MM1. A $60^{\prime \prime} \times 60^{\prime \prime}$ integrated emission map is presented in Figure 4. The map is centered at the reference position and the emission was sampled every $10^{\prime \prime}$ in both $\alpha$ and $\delta$. Also, a short 5 minutes scan was done at the center to increase the signal to noise in the spectrum. The spectrum, shown in Figure 5 with its corresponding Gaussian fit, was binned every 10 channels giving a velocity resolution of 1 
$\mathrm{km} \mathrm{s}^{-1}$. From the fit, we obtained a peak intensity of $2.1 \mathrm{~K}$ with a rms noise of $\sigma=80 \mathrm{mK}$. No hyperfine structure is seen as is likely blended due to a large line-width of $\Delta v=7.5 \mathrm{~km} \mathrm{~s}^{-1}$ seen in the emission, which has also been seen by others (Friesen et al. 2010, for $\mathrm{N}_{2} \mathrm{H}^{+}(4 \rightarrow 3)$ and Fontani et al. 2006, for $\mathrm{N}_{2} \mathrm{H}^{+}(3 \rightarrow 2)$ ). The $\mathrm{N}_{2} \mathrm{H}^{+}$center spectrum seems to be consistent with an optically thick line centered at $v=97.14 \mathrm{~km} \mathrm{~s}^{-1}$, which is blue-shifted from the core $\mathrm{v}_{l s r}$ and in agreement with infalling evidence presented in PCI and by others for similar high mass star forming regions (Fuller et al. 2005). The $\mathrm{N}_{2} \mathrm{H}^{+}$integrated intensity map appears as a filament from South-East to North-West, which is consistent with the ${ }^{12} \mathrm{CO}(3 \rightarrow 2), \operatorname{HCN}(4 \rightarrow 3)$, and $\mathrm{HCO}^{+}(4 \rightarrow 3)$ mapping done in PCI (see Figures 2,5 , and 8 in that work) and our $\mathrm{C}^{18} \mathrm{O}(3 \rightarrow 2)$ map. This filament is also suggested by the continuum emission maps at $1 \mathrm{~mm}$ and $350 \mu \mathrm{m}$, and integrated intensity $\mathrm{HCO}^{+}(3 \rightarrow 2)$ maps of Motte et al. (2003). A common correlation found in all these maps is that the peak integrated emission is coincident with the dust peak, where the dust peak is located at the reference position used here and taken from our previous interferometric observations on this source (Cortes \& Crutcher 2006). However, in our $\mathrm{N}_{2} \mathrm{H}^{+}$map the peak is offset one beam from the reference position. At this frequency, the ASTE beam is around 20" which coincides with the core size inferred from the interferometric data. This suggests that the emission from this point in the map come from independent regions of the MM1 clump, which at the assumed distance of $5.5 \mathrm{kpc}$ corresponding to $0.5 \mathrm{pc}$. Comparing with the $\mathrm{HCO}^{+}$map, we see that the emission is seen enclosing a larger area around the dust peak, which includes the $\mathrm{N}_{2} \mathrm{H}^{+}$peak ( $T \Delta v$ is over $45 \mathrm{~K} \mathrm{~km} \mathrm{~s}^{-1}$ at the innermost contour). The integrated intensity quickly decreases north of the peak in both maps. In our previous maps of $\mathrm{HCN}(4 \rightarrow 3)$ and $\mathrm{CS}(7 \rightarrow 6)$ we saw little or almost no emission offset from the center suggesting that most of the dense neutral gas was unresolved and inside the MM1 core. Even though $\mathrm{CO}$ emission is expected to be widespread, a similar situation is suggested in the $\mathrm{C}^{18} \mathrm{O}$ map where the dust peak coincides with a local $\mathrm{C}^{18} \mathrm{O}$ peak at the same position, quickly decreasing outside the dust peak. Figure 6 shows the $\mathrm{N}_{2} \mathrm{H}^{+}(4 \rightarrow 3)$ spectra towards selected offsets from the reference position corresponding to the 
brightest points in the map. All three spectra have higher intensities than the spectra taken at the center of the map. Similar situations have been in seen in low and high mass star forming regions where $\mathrm{N}_{2} \mathrm{H}^{+}$appears to be depleted towards the dust peaks (Daniel et al. 2007; Pirogov et al. 2007; Zinchenko et al. 2009; Miettinen et al. 2009; Johnstone et al. 2010; Busquet et al. 2011). It is likely that chemical differentiation has depleated $\mathrm{N}_{2} \mathrm{H}^{+}$at the center of MM1.

\section{Discussion}

\subsection{The un-identified line in the $\mathrm{DCO}^{+}$spectrum}

To explore the nature of the un-identified line seen besides the $\operatorname{DCO}^{+}(5 \rightarrow 4)$ spectrum, we first considered the possibility of a spurious instrumental signal. The ASTE telescope has a cartridge-type side-band separating the (2SB) mixer receiver working at the $350 \mathrm{GHz}$ band. The image rejection ratio is $15 \mathrm{~dB}$, which makes opposite sideband leakage unlikely. The telescope receiver has been carefully tested and no spurious signals are reported at that frequency (T. Sakai, priv. comm.). The $\mathrm{DCO}^{+}$line was observed in the upper sideband of the ASTE receiver. Thus, we searched for strong lines in the lower sideband (12 GHz away), which may have leaked into the upper sideband. Leaking from the lower sideband would require a strong line due to the high side-band image rejection ratio and according to the splatalogue database for molecular spectroscopy, no strong lines are present in a $500 \mathrm{MHz}$ interval centered at $348.169 \mathrm{GHz}$. Also, contamination from atmospheric lines is also ruled out as the position switching removes any atmospheric contribution. Another possibility is that the line is the result of a baseline ripple. These instabilities are usually the result of a standing wave leaked, due to reflections between the feed and the antenna structures, into the receiver. A possibility is oscillations of the goretex membrane that covers the receiver cabin under the dish. To explore this possibility, the 484 scans gathered were inspected one by one. Those with clear ripples were flagged-out and not used. After baseline removal, the remaining scans were inspected again looking for further instabilities. 
Only those with flat baselines were kept, which imply removing about $30 \%$ of the observations. However even after removing spectra with ripples, it cannot be ruled out that the line feature is the result of such instability. Having established this, we consider the possibility of the signal being real. If the feature is a molecular transition, its rest frequency would be $360.1847 \mathrm{GHz}$. The closest molecular transition is the ${ }^{13} \mathrm{C}$ isotopologue of formic acid, in the $16(3,14) \rightarrow 15(3,13)$ at $360.18795 \mathrm{GHz}$, as reported by the splatalogue database but originally provided by CDMS (Muller et al. 2005). However, the emission seen here is too strong even for ${ }^{12} \mathrm{C}$ formic acid (Arce et al. 2008) and thus, this possibility is unlikely. On the other hand, there is always the possibility that we are seeing a transition from an un-identified molecule. Another scenario is that this line corresponds to $\mathrm{DCO}^{+}$emission at a different velocity. If that is the case, it will mean strong $\mathrm{DCO}^{+}$emission from a cold component in the line of sight. This would be an exciting new result as deuterium chemistry is thought to be enhanced in cold cores by freezing out of carbon bearing molecules on grain surfaces (Bergin \& Tafalla 2007). Nevertheless, we cannot conclude with certainty what the nature of the emission seen here is. It is difficult to correlate with emission from other molecules as their line-width is large, specially HCN. Only more independent observations will help confirming this un-identified emission.

\subsection{Column densities and abundances}

Assuming that the clump is in general homogeneous and isothermal, it is possible to obtain information about the optical depths and column densities by analyzing the line intensities and the velocity dispersions. Based on these assumptions, we used the radiative transfer code RADEX (Schöier et al. 2005) to estimate the column densities and abundances for several molecules including some of the ones presented in PCI (see Table 2). The detailed source geometry is not known as the core is unresolved and the number of sources inside MM1 is yet to be determined. Thus, we make no assumptions about the clump structure or possible number of sources inside. 
As the RADEX code does not assume any kind of source geometry or velocity fields, the column density for the emitting material is adjusted until the line intensity is matched. We also corrected for beam dilution by multiplying the output from RADEX by the beam filling factor calculated for each transition where the source size was obtained from the interferometric observations of Cortes \& Crutcher (2006). Because the beam sizes for all molecules are comparable (differences are $\sim 2^{\prime \prime}$, see Table 1), the correction is small. Thus we should not expect strong beam-diluted emission if the molecular gas follows the dust distribution. However, there is evidence for a remarkably warm hot-core inside the MM1 clump with $\mathrm{T} \sim 400 \mathrm{~K}$ and $0.03 \mathrm{pc}$ in size (Sridharan to be submitted 2011). As the MM1 hot core is very small in size, any emission coming only from the hot core will be extremely diluted within any current single dish beam.

We derived column densities for all observed molecules, with the exception of $\mathrm{HCO}^{+}$and $\mathrm{HCN}$ as the lines are self-absorbed and more accurate geometric source modeling for the radiative transfer problem should be done. Instead, we used RADEX for the less abundant $\mathrm{H}^{13} \mathrm{CO}^{+}$and $\mathrm{H}^{13} \mathrm{CN}$ to estimate their column densities and then used a ${ }^{12} \mathrm{C} /{ }^{13} \mathrm{C}$ ratio of 50 as observed in the molecular ring (Wilson \& Rood 1994) to derive the column densities for the main isotopomers. The $\mathrm{N}_{2} \mathrm{H}^{+}$column density was derived using the collision rates provided by RADEX where hyperfine structure is considered in the calculation. However, the hyperfine structure appears to be blended in the main emission as the line-width is larger than the hyperfine components separation. The remaining molecular column densities where calculated using RADEX and then were compared with classical column density estimations (Caselli et al. 2002b), agreement was within a factor of 2 .

To estimate abundances across the MM1 main axis, we chose 3 pointings from north-east to south-west across MM1 main axis, including the center of MM1. The pointings are indicated as crosses in the $\mathrm{C}^{18} \mathrm{O}$ map (see Figure 1). Thus, we derived column densities along the filament for all available molecules with the exception of $\mathrm{DCO}^{+}$which has only 1 data point and $\mathrm{CS}$ which 
does not show emission over the $3 \sigma$ level at the $\left(30^{\prime \prime}, 30^{\prime \prime}\right)$ relative offset. The column density from the dust map obtained by Cortes \& Crutcher (2006) was used for the center pointing and new values were derived from the $350 \mu \mathrm{m}$ fluxes reported by Motte et al. (2003) for the remaining positions. The hydrogen column densities were derived using

$$
\left(N_{\mathrm{H}} / \mathrm{cm}^{-2}\right)=1.93 \times 10^{15} \frac{\left(S_{v} / \mathrm{Jy}\right) \lambda_{\mu \mathrm{m}}^{4}}{\left(\theta_{s} / \operatorname{arcsec}\right)^{2}\left(Z / Z_{\odot}\right) b T} \frac{e^{x}-1}{x}
$$

where $S_{v}$ is the averaged densitydensity flux from the source in Jy, $\theta_{s}=\sqrt{\theta_{s, \min } \times \theta_{s, \max }}$ is the angular source size, $\left(Z / Z_{\odot}=1\right)$ is relative metalicity to solar neighborhood, $b$ is a parameter that reflects the variation of dust absorption cross sections (Mezger et al. 1990). Two values are used for $b ; b=1.9$ reproduces estimates of $\sigma_{400 \mu m} \sim 8.3 \times 10^{-26} \mathrm{~cm}^{2}(\mathrm{H} \text {-atom })^{-1}$, which represents molecular gas of moderate density $n\left(\mathrm{H}_{2}\right)<10^{6} \mathrm{~cm}^{-3}$, and $b=3.4$ for dust around deeply embedded IR sources at higher densities. We used a value of $b=3.4$ in our estimates for MM1. A value of $T=19 \mathrm{~K}$ is used for the dust temperature derived from the SED fitting by Motte et al. (2003), and $x=\frac{1.44 \times 10^{4}}{\lambda_{\mu m} T}$ is the $\frac{h c}{\lambda k T}$ factor for the Planck function. The hydrogen column densities for the offsets are listed in Table 3 and also indicated as crossed in the $\mathrm{C}^{18} \mathrm{O}$ map in Figure 1, The derived abundances for all molecules are listed in Table 2 and plotted in Figure 7.

\subsection{Comparison with chemical models}

\subsubsection{HCN abundance}

The relative differences between molecular abundances in star forming regions can provide information about the evolutionary state of that region. Chemical differentiation, like the $\mathrm{N} / \mathrm{O}$ ratio, seems to be ubiquitous in high mass star formation, possibly an indication of the evolutionary state of a region (Rodgers \& Charnley 2001; Caselli et al. 2002b; Doty et al. 2002; Rodgers \& Charnley 2003; Roueff et al. 2007). Besides $\mathrm{C}^{18} \mathrm{O}$, the most abundant molecule in 
our sample at the center of the MM1 clump appears to be $\mathrm{HCN}$ followed by $\mathrm{CS}, \mathrm{HCO}^{+}, \mathrm{N}_{2} \mathrm{H}^{+}$, and $\mathrm{DCO}^{+}$. The difference between $\mathrm{CS}$ and $\mathrm{HCN}$ is likely within the calibration error, but larger for the remaining molecules. Jørgensen et al. (2004) studied a sample of low mass and high mass dense cores and found the $\mathrm{HCO}^{+}$abundance comparable to $\mathrm{HCN}$, but lower than the CS abundance in low-mass class I sources while in the high mass case HCN seems to be a factor of 5 larger than $\mathrm{HCO}^{+}$but similar to the CS abundance. A similar situation is seen by Pirogov et al. (2007) in a study towards a sample of southern high mass star forming regions. Due to its high dipole moment, the HCN molecule traces high density gas; its critical density for the $J=4 \rightarrow 3$ transition is $n_{\text {crit }} \sim 10^{7} \mathrm{~cm}^{-3}$. Thus, it is expected to find $\mathrm{HCN}(4 \rightarrow 3)$ excited in dense clumps such as MM1. As the HCN molecule is most likely formed in the gas phase rather than a product of ice mantle evaporation (Rodgers \& Charnley 2001), its abundance might indicate how evolved the clump is. It has been suggested that $\mathrm{NH}_{3}$ would be a major component of ice mantles and within the hot core region its release to the gas-phase will significantly affect the abundance of molecules such as $\mathrm{HCN}$. As a daughter molecule, $\mathrm{HCN}$ is formed by dissociative recombination of $\mathrm{HCNH}^{+}$which is previously formed by recombination of $\mathrm{C}^{+}$with $\mathrm{NH}_{3}$. This scenario is plausible for higher gas temperatures (Rodgers \& Charnley 2001) for models with $T \sim 300 \mathrm{~K}$ in a hot core. Similar models have been produced by Doty et al. (2002) where they find an enhanced HCN abundance as the hot core evolves with time, but using $\mathrm{H}_{2} \mathrm{NC}^{+}$as the favored product to explain the $\mathrm{HCN}$ abundance. In principle the evaporation of ammonia from ice mantles will reduce the abundance of O-bearing molecules and enhance the abundance of N-bearing species. Thus according to the previous studies, our derived abundance of $\mathrm{HCN}$ at the center give a possible age for the MM1 hot core of $10^{4} \mathrm{yr}$. 


\subsubsection{CS abundance}

The abundance of CS in evolved high mass clumps is likely dependent on the evaporation of sulphur from ice mantles by radiation from the central star. As most of the sulphur ejected from dust grains is likely locked into $\mathrm{H}_{2} \mathrm{~S}$ (Doty et al. 2002; Rodgers \& Charnley 2003), or atomic ionized sulphur (Wakelam et al. 2004), this will lead to an increase of the $\mathrm{SO}_{2}$ abundance, which will lead to an increase in the CS abundance. Our derived value for the CS abundance towards the center of MM1 is consistent with both models which place the CS abundance around $10^{-8}$ for an evolutionary time scale of $10^{4} \mathrm{yr}$. However, the main issue behind using sulphur as a chemical clock is the uncertainty regarding the progenitors of S-bearing molecules which is still under debate.

\subsubsection{Deuterium fractionation}

It is known that starless low mass cores have enhanced abundances of deuterium bearing molecules. Due to freezing of carbon and oxygen on grain surfaces, the deuterium abundance can be enhanced by orders of magnitude with respect to more evolved regions (Bergin \& Tafalla 2007). Even though the situation in high mass clumps is not as clear, it is expected that infrared dark clouds will show the same increase in deuterium abundance. A useful parameter to quantify the importance of deuterium in clouds is the deuterium fractionation. This parameter is the ratio between the D-molecule and the $\mathrm{H}$-molecule column densities and can be used to test the evolutionary stage of star forming regions. Thus, we calculated $\mathrm{R}=\left[\mathrm{DCO}^{+}\right] /\left[\mathrm{HCO}^{+}\right]=0.0012$ for the center of MM1. Fractionation values have been calculated for low mass class 0 and class I cores by Roberts \& Millar (2000); Turner (2001); Caselli et al. (2002b) who found values larger than 0.01 with most of the results about 0.06 . Higher fractionation values based on deuterated formaldehyde, about 0.1, have been obtained by Bergman et al. (2011) towards $\rho$-Ophiuchi. In the case of high mass cores, results are not as numerous. Miettinen et al. (2009) found 
moderate deuteration of about 0.03 to 0.04 for Orion B9; while Chen et al. (2010) found moderate deuteration between 0.01 to 0.05 toward the infrared dark cloud G28.34+0.06. Roueff et al. (2007) modeled deuterium fractionation in warm dense clumps, such as MM1, and found that $\left[\mathrm{DCO}^{+}\right] /\left[\mathrm{HCO}^{+}\right]$decreases rapidly with temperature giving fractionation values similar to our findings for $\mathrm{T}<30 \mathrm{~K}$, which suggests that MM1 might be more evolved than previously thought. Considering that infall is proceeding, as showed by PCI, and a hot core has already formed, it is possible that gas in the MM1 envelope has been heated by the hot core to the point where the dust and the gas are no longer in LTE. At the same time, it is also possible that the dust emission is optically thick. In both cases the dust temperature is not representative of the real kinetic gas temperature inside the MM1 clump. Even though the amount of deuteration found in MM1 is not significant as in pre-stellar sources, it is still significant for a warm environment such as the one suggested by the model. The $\mathrm{DCO}^{+}$molecule can be assembled by combination of $\mathrm{CO}$ with $\mathrm{H}_{2} \mathrm{D}^{+}$ which is very efficient at low temperatures. However, chemical modeling by Roberts \& Millar (2000) suggests that this mechanism is no longer efficient when temperature increases, which is also suggested by Roueff et al. (2007). As the envelope gas temperature rises the relative abundance of $\mathrm{C}, \mathrm{N}$, and $\mathrm{O}$ molecules tend to decrease the abundance of $\mathrm{H}_{2} \mathrm{D}^{+}$which can no longer efficiently combine with $\mathrm{CO}$ to produce $\mathrm{DCO}^{+}$. Instead, $\mathrm{CH}_{2} \mathrm{D}^{+}$might become the dominant pathway for $\mathrm{DCO}^{+}$creation by $\mathrm{CH}_{2} \mathrm{D}^{+}+\mathrm{O} \rightarrow \mathrm{DCO}^{+}+\mathrm{H}_{2}$ as the deuteration of $\mathrm{CH}_{3}^{+}$can proceed to much higher levels than $\mathrm{H}_{2} \mathrm{D}^{+}$, the usual way to produce $\mathrm{DCO}^{+}$(A. Wootten, priv. comm.). As previously mentioned, we question how well the derived temperature from the SED represents the real envelope gas temperature; it could well be warmer than the dust as the dust may have become optically thick. So it is possible that the $\mathrm{DCO}^{+}$emission that we are seeing comes from a different chemical pathway than what is seen in colder sources. 


\subsection{4. $\mathrm{N}_{2} \mathrm{H}^{+}$and $\mathrm{HCO}^{+}$abundances}

For high mass star forming regions, $\mathrm{HCO}^{+}$abundances have been derived from a sample of sources similar to MM1 with values in the $10^{-11}$ to $10^{-10}$ range (Zinchenko et al. 2009). $\mathrm{H}^{13} \mathrm{CO}^{+}$ column densities have been derived as well, toward several high mass cores at different stages of evolution with values that range between $10^{12}$ to $10^{13} \mathrm{~cm}^{-2}$ (Szymczak et al. 2007; Sakai et al. 2010). Our derived $\mathrm{HCO}^{+}$abundance towards the center of the $\mathrm{MM} 1$ clump is $X\left(\mathrm{HCO}^{+}\right)=$ $1.5 \times 10^{-11}$ and the $\mathrm{H}^{13} \mathrm{CO}^{+}$column density is $2 \times 10^{13}$ which are consistent with results in similar regions and with the chemical models previously described. The $\mathrm{N}_{2} \mathrm{H}^{+}$abundance has also been derived towards high mass cores, mostly from $\mathrm{N}_{2} \mathrm{H}^{+}(1 \rightarrow 0)$ observations as there are few $\mathrm{N}_{2} \mathrm{H}^{+}(4 \rightarrow 3)$ results in the literature. Current findings yield abundances between $10^{-11}$ to 10 ${ }^{-10}$ (Daniel et al. 2007; Pirogov et al. 2007; Zinchenko et al. 2009; Miettinen et al. 2009). Our derived abundance towards the center of MM1 is $X\left(\mathrm{~N}_{2} \mathrm{H}^{+}\right)=9.6 \times 10^{-11}$ which is consistent with previous results. Busquet et al. (2011) produced a chemical model for the high mass star forming region AFGL 5142. They modeled the effect of a central source on the abundance of several species including $\mathrm{N}_{2} \mathrm{H}^{+}$. At an evolutionary time of $10^{4} \mathrm{yr}$, their model predicts an abundance of $X\left(\mathrm{~N}_{2} \mathrm{H}^{+}\right) \sim 10^{-10}$ for the central core of AFGL 5142, which is about the abundance value that we find for MM1 and in the same order of magnitude with the age obtained previously by comparing $\mathrm{HCN}, \mathrm{CS}$, and $\mathrm{DCO}^{+}$abundances with other chemical models. Lintott et al. (2005) compared the production of $\mathrm{N}_{2} \mathrm{H}^{+}$and related chemical species for high mass star formation environments. While they do not present abundances for $\mathrm{N}_{2} \mathrm{H}^{+}$, they studied the relative abundance between CS and $\mathrm{N}_{2} \mathrm{H}^{+}$which we can compare here. Their model predicts that accelerated infall will produce a differentiation between $\mathrm{CS}, \mathrm{HCN}$, and $\mathrm{N}_{2} \mathrm{H}^{+}$, where they expect that relative abundances will decrease due to the destruction of $\mathrm{N}_{2} \mathrm{H}^{+}$by $\mathrm{CO}$ and enhancement of $\mathrm{CS}$ by the accelerated collapse. At the center of MM1 we obtained a $\log \left([\mathrm{CS}] /\left[\mathrm{N}_{2} \mathrm{H}^{+}\right]\right)=2.3$, which in the Lintott et al. (2005) model is close to no acceleration scenario. As MM1 is infalling with speed slightly supersonic but sub-alfvenic (see section 6.6), it is difficult to reconcile this with a no acceleration 
model. However, we do see an enhancement of the CS abundance at the center of MM1 which will be discussed in the following section. 
Table 2: Column densities and abundances are presented here.

\begin{tabular}{ccccc}
\hline \hline Molecule & $\begin{array}{c}\text { Offsets } \\
{[(\operatorname{arcsec}, \operatorname{arcsec})]}\end{array}$ & $\begin{array}{c}\text { Column Density } \\
{\left[\mathrm{cm}^{-2}\right]}\end{array}$ & Abundance & $\begin{array}{c}\text { Line-width } \\
\mathrm{km} \mathrm{s}^{-1}\end{array}$ \\
\hline $\mathrm{DCO}^{+}$ & $(0,0)$ & $1.2 \times 10^{12}$ & $8.8 \times 10^{-13}$ & 2.9 \\
$\mathrm{~N}_{2} \mathrm{H}^{+}$ & $(0,0)$ & $1.3 \times 10^{14}$ & $9.6 \times 10^{-11}$ & 7.3 \\
$\mathrm{~N}_{2} \mathrm{H}^{+}$ & $(30,30)$ & $6.0 \times 10^{13}$ & $1.1 \times 10^{-10}$ & 6.3 \\
$\mathrm{~N}_{2} \mathrm{H}^{+}$ & $(-30,-15)$ & $1.3 \times 10^{14}$ & $2.0 \times 10^{-10}$ & 5.6 \\
$\mathrm{HCO}^{+}$ & $(0,0)$ & $1.0 \times 10^{15}$ & $7.4 \times 10^{-10}$ & 8.4 \\
$\mathrm{HCO}^{+}$ & $(30,30)$ & $2.5 \times 10^{14}$ & $7.9 \times 10^{-10}$ & 6.2 \\
$\mathrm{HCO}^{+}$ & $(-30,-15)$ & $1.5 \times 10^{14}$ & $2.4 \times 10^{-10}$ & 6.2 \\
$\mathrm{H}^{13} \mathrm{CO}^{+}$ & $(0,0)$ & $2.0 \times 10^{13}$ & $1.5 \times 10^{-11}$ & 3.0 \\
$\mathrm{HCN}^{+}$ & $(0,0)$ & $2.0 \times 10^{16}$ & $1.5 \times 10^{-8}$ & 9.5 \\
$\mathrm{HCN}$ & $(30,30)$ & $8.0 \times 10^{14}$ & $2.5 \times 10^{-9}$ & 7.6 \\
$\mathrm{HCN}$ & $(-30,-15)$ & $2.5 \times 10^{15}$ & $4.0 \times 10^{-9}$ & 14 \\
$\mathrm{H}^{13} \mathrm{CN}$ & $(0,0)$ & $4.0 \times 10^{14}$ & $2.9 \times 10^{-10}$ & 4.8 \\
$\mathrm{CS}$ & $(0,0)$ & $1.0 \times 10^{16}$ & $7.3 \times 10^{-9}$ & 4.3 \\
$\mathrm{CS}$ & $(-30,-15)$ & $1.3 \times 10^{15}$ & $2.1 \times 10^{-9}$ & 9.4 \\
$\mathrm{C}^{18} \mathrm{O}$ & $(0,0)$ & $2.9 \times 10^{16}$ & $2.1 \times 10^{-8}$ & 6.4 \\
$\mathrm{C}^{18} \mathrm{O}$ & $(30,30)$ & $8.0 \times 10^{15}$ & $2.5 \times 10^{-8}$ & 5.1 \\
$\mathrm{C}^{18} \mathrm{O}$ & $(-30,-15)$ & $1.1 \times 10^{16}$ & $1.7 \times 10^{-8}$ & 5.7 \\
\hline
\end{tabular}


Table 3: Hydrogen column densities for three selected pointings are shown here.

\begin{tabular}{cc}
\hline \hline Offsets $(\alpha, \delta)$ & Column Density \\
$([\operatorname{arcsec}],[\operatorname{arcsec}])$ & {$\left[\mathrm{cm}^{-2}\right]$} \\
\hline$(-30,-15)$ & $6.3 \times 10^{23}$ \\
$(0,0)$ & $1.5 \times 10^{24}$ \\
$(30,30)$ & $3.2 \times 10^{23}$ \\
\hline
\end{tabular}




\subsection{Spatial differentiation}

In all the maps produced from our observations, we see evidence for a filament in the MM1 clump (see also PCI and Motte et al. (2003)). The $\mathrm{C}^{18} \mathrm{O}$ integrated emission map is consistent with this, as well as our abundance derivation which is constant along the main axis of the filament. The spatial distribution of both ${ }^{12} \mathrm{CO}$ and dust (both 850 and $350 \mu \mathrm{m}$ ) also show a single filament with a continuous progression in the emission from the MM1 center to the end of the filament at the south-east edge. The ${ }^{12} \mathrm{CO}$ map however, shows strong peaks in MM1 and at the south-east edge where there is a likely boundary with the H II region. Only the MM1 center peak is seen in dust emission maps. Our $\mathrm{C}^{18} \mathrm{O}$ map shows two distinct areas with strong emission around the dust peak and close to the south-east boundary. There is a clear region in the middle where the emission drops in intensity, which is not seen in the dust maps and it is not completely clear in the ${ }^{12} \mathrm{CO}$ map. This suggests that the MM1 filament might not extend completely to the boundary with the $\mathrm{H}$ II region as $\mathrm{C}^{18} \mathrm{O}$ is likely showing us the integrated emission through the cloud rather than just the envelope. The influence of H II region ionization and shock fronts as external pressure over the MM1 clumps is important as a mechanism for triggered star formation. The $\mathrm{N}_{2} \mathrm{H}^{+}$emission is also distributed like $\mathrm{C}^{18} \mathrm{O}$ and $\mathrm{HCO}^{+}$, but we noticed that the peak emission in $\mathrm{N}_{2} \mathrm{H}^{+}$is not coincident with the dust peak as seen with the other molecules. The main peak in the $\mathrm{N}_{2} \mathrm{H}^{+}$integrated emission is 1 beam away from the center to $\left(0^{\prime \prime}, 20^{\prime \prime}\right)$. Thus, it cannot be attributed to an oversampled grid re-distributing emission. Caselli et al. (2002b) suggested that $\mathrm{N}_{2} \mathrm{H}^{+}$followed the dust emission in cold cores as it has been seen that $\mathrm{N}$-bearing species do not get frozen on dust grains until densities reach the $10^{5}$ to $10^{6}$ range with gas temperatures around

$10 \mathrm{~K}$ (Bergin \& Tafalla 2007). However, when the gas gets warmer due to heating from the central source(s), $\mathrm{CO}$ is rapidly released from grain ice mantles quickly destroying $\mathrm{N}_{2} \mathrm{H}^{+}$. If the gas in MM1 is being warmed by the hot core releasing $\mathrm{CO}$ into the gas phase, it may explain the offset seen in the $\mathrm{N}_{2} \mathrm{H}^{+}$peak emission with respect to the dust peak. As our sub-millimeter observations 
trace higher critical densities, it is likely that we are seeing gas closer to the hot core where the temperatures are likely higher than the dust SED suggested.

We also noticed that the emission from the neutral molecules is more compact than the ions, with the exception of the $\mathrm{H}^{13} \mathrm{CO}^{+}$and $\mathrm{H}^{13} \mathrm{CN}$ which only have significant emission from the center of MM1. The HCN and CS emission are mostly confined to the clump, where CS is remarkably compact (see PCI Figures 7 and 8). But, the $\mathrm{N}_{2} \mathrm{H}^{+}$and $\mathrm{HCO}^{+}$integrated emission are much more widespread over the filament. All these molecules have similar critical densities for the rotational transitions observed; thus, they presumably come from gas under the same physical conditions. In order to explore possible explanations for this spatial differentiation, we will consider the effect that the magnetic field threading MM1 might have over the gas along the filament. If the field is frozen in the ions, it is likely that the tension created by the field will limit the movement of ions along the filament; while the neutrals can diffuse more freely through the ions. Table 2 shows the line-widths obtained by adjusting Gaussian profiles to the line emission at the selected positions along the strip. It is evident that the line-width of $\mathrm{N}_{2} \mathrm{H}^{+}$and $\mathrm{HCO}^{+}$are consistently smaller than the neutral molecule line-widths. Particularly at the center we also have $\mathrm{DCO}^{+}$and the ${ }^{13} \mathrm{C}$ isotopomers of $\mathrm{HCN}$ and $\mathrm{HCO}^{+}$. As these species are optically thin their velocity dispersions are more representative than their more abundant ${ }^{12} \mathrm{C}$ isotopomers. All ions show smaller velocity dispersions than neutrals with $\mathrm{DCO}^{+}$and $\mathrm{H}^{13} \mathrm{CO}^{+}$about $3.0 \mathrm{~km} \mathrm{~s}^{-1} \mathrm{FWHM}$; while $\mathrm{H}^{13} \mathrm{CN}$ has the largest line-width at $4.8 \mathrm{~km} \mathrm{~s}^{-1}$. It has already been noted by Houde et al. (2000a,b) that ions should have smaller velocity dispersion due to trapping on magnetic field lines. Li et al. (2010) proposed that the smaller velocity dispersion of ions with respect to neutrals, is a signature for turbulent ambipolar diffusion ( $\mathrm{Li}$ et al. 2010). While ambipolar diffusion is inevitable at some point during the clump evolution, how fast the neutrals will diffuse is still a matter of debate (McKee \& Ostriker 2007).

Chemical models of dense clumps, including inner hot cores, have calculated radial 
profiles for the abundance of different molecules (Doty et al. 2002; Rodgers \& Charnley 2003; Nomura \& Millar 2004). All these models find that ion abundances increase rapidly with distance from the central source(s). Unfortunately, not all these models present results for all the molecules presented here. Thus, we will concentrate on comparing with the model that presents the largest number of molecular abundances. Rodgers \& Charnley (2003) produced models for envelopes surrounding hot cores, as we believe is the MM1 case. They developed models based on the dynamical state of the envelope, considering static and collapsing cases (unfortunately without including magnetic fields). For their collapse model and assuming gas temperatures of 300-400 K for the hot core, they found $\mathrm{HCO}^{+}$abundances up to a few tenths of $10^{-9}$ at $1 \mathrm{pc}$ from the central source(s) for $10^{4} \mathrm{yr}$ and $10^{-8}$ for $10^{5} \mathrm{yr}$ of evolution after collapse started. As PCI presented compelling evidence of infall in MM1, comparing with this model seems pertinent. Even though the UMIST database includes $\mathrm{N}_{2} \mathrm{H}^{+}$, they did not present predictions for that molecule in their work. Table 2 shows $\mathrm{HCO}^{+}$column densities and abundances at distances of about $1 \mathrm{pc}$ from the center at both sides of the filament. Our values are in the range of $10^{-10}$ to $10^{-11}$ which is at least an order of magnitude lower than the Rodgers \& Charnley (2003) model. The $\mathrm{HCO}^{+}$ abundance is derived from $\mathrm{H}^{13} \mathrm{CO}^{+}$, which is moderately optically thin (from the RADEX results, $\tau=0.7)$, but we assumed a ${ }^{12} \mathrm{CO} /{ }^{13} \mathrm{CO}$ ratio of 50 which is not necessarily accurate. However even using values close to 70 for the ${ }^{12} \mathrm{CO} /{ }^{13} \mathrm{CO}$ ratio, the $\mathrm{HCO}^{+}$abundance will not agree with the prediction. Interestingly the Rodgers \& Charnley (2003) model predicts a sudden decrease in the abundance of neutral molecules such as CS, with a cutoff point around the collapse front. The case of $\mathrm{HCN}$ is not as clear, as their models show a small decrease in abundance at the collapse front, but an increase towards the envelope. The HCN abundance predicted at $1 \mathrm{pc}$ from the center is about $5 \times 10^{-9}$, which is about what we see from our data. For CS we derived an abundance similar to $\mathrm{HCN}$ at $1 \mathrm{pc}$, but the situation is not symmetric as we do not have emission at the other end. This CS emission at offset $\left(-30^{\prime \prime},-15^{\prime \prime}\right)$ is likely coming from the same place where we see additional HCN emission ( see Figure 7 in PCI); thus, it would not be a valid comparison with 
the model. However, we do know that the CS emission in MM1 is fairly compact which is also predicted by Rodgers \& Charnley (2003). The overall picture presented by the model is similar to what we have determined from our observations, particularly with the strong CS cutoff at the collapse front. Additionally, the panel presented by PCI for HCN infalling spectra places the collapse front at $25^{\prime \prime}$ in radius (or about $0.9 \mathrm{pc}$ ) which is also similar to the Rodgers \& Charnley (2003) results. Even tough our abundances for neutral molecules agree with that model, the $\mathrm{HCO}^{+}$ molecule does not. However, it is feasible that changes in the chemical abundances due to the heating of the hot core explains the spatial differentiation that we are seeing in the MM1 clump.

\subsection{The ionization rate}

\subsubsection{The electron fraction}

The abundance of molecular ions depends on a complicated interplay between gas-phase chemistry and cosmic-ray driven chemistry. Molecular ion abundances are critical to understand the interaction between the magnetic field and the neutral gas as the magnetic field can only influence the dynamics of dense neutral gas through the ions. The electron fraction is estimated by charge equilibrium between the electrons and the most abundant ions in a molecular clump as we assume overall charge neutrality. The electron fraction permits calculations of neutral-ion coupling parameters, which we can use to understand the impact of the magnetic field in the gas dynamics of the infalling MM1 clump. As $\mathrm{H}_{3}^{+}, \mathrm{H}_{2} \mathrm{D}^{+}, \mathrm{DCO}^{+}, \mathrm{HCO}^{+}, \mathrm{N}_{2} \mathrm{H}^{+}$, and $\mathrm{N}_{2} \mathrm{D}^{+}$are considered the most abundant ions in a high mass clump (Miettinen et al. 2009; Caselli et al. 2008, 2002b; Bergin et al. 1999; Williams et al. 1998; Wootten et al. 1979), a lower limit for X(e) can be written as

$$
\mathrm{X}(\mathrm{e}) \geq \mathrm{X}\left(\mathrm{H}_{3}^{+}\right)+\mathrm{X}\left(\mathrm{H}_{2} \mathrm{D}^{+}\right)+\mathrm{X}\left(\mathrm{DCO}^{+}\right)+\mathrm{X}\left(\mathrm{HCO}^{+}\right)+\mathrm{X}\left(\mathrm{N}_{2} \mathrm{H}^{+}\right)+\mathrm{X}\left(\mathrm{N}_{2} \mathrm{D}^{+}\right)
$$


As previously shown, the deuterium fractionation is not large in MM1 as the clump has already evolved from a pre-stellar phase. This suggests that deuterium species, such as $\mathrm{N}_{2} \mathrm{D}^{+}$and $\mathrm{DCO}^{+}$, are not directly important in the derivation of the electron fraction and can be neglected in the calculation. The case of $\mathrm{H}_{2} \mathrm{D}^{+}$is somewhat different, as it is directly related to the $\mathrm{H}_{3}^{+}$ abundance through $\mathrm{H}_{3}^{+}+\mathrm{HD} \leftrightarrow \mathrm{H}_{2} \mathrm{D}^{+}+\mathrm{H}_{2}$, and thus cannot be neglected from equation 2 . The abundance of $\mathrm{H}_{2} \mathrm{D}^{+}$can be estimated from the survey of Caselli et al. (2008) towards a sample of star forming dense cores. In that work, the abundance of $\mathrm{H}_{2} \mathrm{D}^{+}$was calculated by observing the ortho- $\mathrm{H}_{2} \mathrm{D}^{+}\left(1_{1,0}-1_{1,1}\right)$ line with $\mathrm{CSO} 4$. To obtain an estimate of the $\mathrm{H}_{2} \mathrm{D}^{+}$abundance, we used an average of their values toward dense cores, or $X\left(\mathrm{H}_{2} \mathrm{D}^{+}\right)=2.3 \times 10^{-10}$, and assumed an ortho-to-para ratio of $10^{-4}$ as used by Miettinen et al. (2009). We are here assuming an average of the abundance values derived by Caselli et al. (2008) are representative for MM1. Some dense cores used by them are indeed similar to MM1 (such as Ori B9), so using an average is a compromise for a better representation of $\mathrm{H}_{2} \mathrm{D}^{+}$abundance in star forming regions. Now, the deuterium fraction $R$ provides a rough estimate of the $r=\left[\mathrm{H}_{2} \mathrm{D}^{+}\right] /\left[\mathrm{H}_{3}^{+}\right]$abundance ratio through the relation derived by Crapsi et al. (2004), $R=\left(r+2 r^{2}\right) /\left(3+2 r+r^{2}\right)$, by assuming a simplified chemical network for deuterium in molecular clouds. Thus, by numerically solving the Crapsi et al. (2004) relation and using $R=\left[\mathrm{DCO}^{+}\right] /\left[\mathrm{HCO}^{+}\right]=1.2 \times 10^{-3}$ from our observations, we obtain $X\left(\mathrm{H}_{3}^{+}\right)=6.4 \times 10^{-8}$ giving an electron fraction of $\mathrm{X}(\mathrm{e})=6.5 \times 10^{-8}$. Our estimation for the electron fraction is consistent with other high mass star forming sites such as Ori 9 where Miettinen et al. (2009) calculated $6 \times 10^{-7}$ and DR21(OH) where Hezareh et al. (2008) obtained $3.2 \times 10^{-8}$, and in the upper range of low mass cores such as L1544 where Caselli et al. (2002b) found $\mathrm{X}(\mathrm{e}) \sim 10^{-9}$.

\footnotetext{
${ }^{4}$ The CSO has a similar beam size as our ASTE observations
} 


\subsection{The Ion-Neutral coupling}

The effect of a magnetic field in a weakly ionized gas depends on how well the neutrals are coupled to the ions. A well coupled field-gas will be more efficient in restraining the neutrals movement in directions parallel to the field where magnetic support does not directly delay the gas infall. We first noticed that infall in MM1 is slightly supersonic but sub-alfvenic, where $\mathrm{v}_{\text {if }}=0.5 \mathrm{~km} \mathrm{~s}^{-1}$ is the infalling speed, $\mathrm{C}_{\mathrm{s}}=0.46 \mathrm{~km} \mathrm{~s}^{-1}$ is the sound speed, and $\mathrm{v}_{\mathrm{A}}=2.4 \mathrm{~km} \mathrm{~s}^{-1}$ is the Alfven speed. These parameters yield a Mach number of $M=1.1$ and Alfvenic Mach number of $\mathrm{M}_{\mathrm{A}}=0.2$. As the infall is sub-alfvenic, $\mathrm{MHD}$ waves can in principle propagate faster than the collapsing gas. If the gas is warmer than the dust as previously suggested, the sound speed will be larger making the infall sub-sonic.

It has been found in laboratory plasmas that magnetic Reynolds numbers much larger than 1 suggests that coupling between the gas and the field is strong and in principle, this allows the gas and the field to move as a single system. This number is defined as the ratio between the inertial term to the viscous term in a magnetized fluid, or $R_{m}=\frac{\mathrm{v}_{\text {if }} l}{v_{M}}$, where $\mathrm{v}_{\text {if }}$ is the fluid speed, $l$ is the length scale in which the magnetic field has a significant change in magnitude, and $v_{M}$ is the magnetic viscosity defined as $v_{M}=\mathrm{v}_{\mathrm{A}}^{2} / v_{i n}$, where $v_{i n}$ is the ion-neutral collision frequency defined as $v_{i n}=X(e) n_{\mathrm{H}_{2}}<\sigma_{i n} v>$, where $\left\langle\sigma_{i n} v>\right.$ is the ion-neutral collision rate. Thus, we calculate the magnetic (ambipolar diffusion) Reynolds number as $R_{m}=\frac{\mathrm{v}_{\mathrm{if}} l}{\mathrm{v}_{\mathrm{A}}^{2}} X(e) n_{H}<\sigma_{i n} v>$, where $l$ is calculated to be about $\sim 5^{\prime \prime}$ or $\sim 0.1 \mathrm{pc}$ obtained from the dust polarization map of Cortes \& Crutcher (2006). The Alfven speed is $\mathrm{v}_{\mathrm{A}}=2.2 \times 10^{5} \mathrm{~B} /\left(n_{H} A_{n}\right)^{1 / 2}$ where we take $A_{n}=1.4$ for representative molecular gas of cosmic abundance (Zweibel 2002), $n_{H}=4.5 \times 10^{5}$ $\mathrm{cm}^{-3}$ from Cortes \& Crutcher (2006), and $B=855 \mu \mathrm{G}$ from PCI. The $\left\langle\sigma_{i n} v>\right.$ is the ion-neutral collision rate which is calculated using the Langeving approximation for the polarization potential (McDaniel \& Mason 1973). Here, we use a value of $\left\langle\sigma_{\text {in }} v\right\rangle=1.69 \times 10^{-9} \mathrm{~cm}^{3} \mathrm{~s}^{-1}$ for molecular gas in a typical star forming region (Mouschovias \& Paleologou 1981). Thus by using our derived 
values for $X(e)$, we calculate $R_{m}=18.1$. As mentioned before, the ambipolar diffusion Reynolds number describes the stability of a magnetized flow, or how close the fluid is to become turbulent. The boundary, or critical point, is often found for astrophysical fluid plasmas comparable to the Hartmann number $M$ (Myers \& Khersonsky 1995), which is defined as the ratio of the Lorentz force to the viscous forces (Davidson 2001) and it can be written as $M=B l \sqrt{\sigma / \eta}$, where $\sigma=c^{2} /\left(4 \pi v_{M}\right)$ is the electrical conductivity and $\eta=\rho v$ is the dynamic viscosity function of the kinematic viscosity $v$. Myers \& Khersonsky (1995) derived the Hartmann number as a function of column density as $M=1.99 \times 10^{7}\left(N / 10^{21} \mathrm{~cm}^{-2}\right) X(e)^{1 / 2}$, the expression that we use here. In astrophysical weakly ionized plasmas, if $R_{m} \gg M \gg 1$ the flow and the field can be considered to be turbulent as the dissipative term is not large enough to damp the inertial term, but if $M>R_{m} \gg 1$ the field can still damp the turbulence in the flow stopping the cascade into smaller eddies. Thus, we directly calculate the Hartmann number finding $M=7 \times 10^{6}$. This value is consistent with calculations of Myers \& Khersonsky (1995) towards similar dense cores and to recent numerical simulations of sub-alfvenic flows (Li et al. 2008; McKee et al. 2010).

The ratio between the core size, $R$, and the minimum MHD wavelength, or cutoff wavelenght, for propagation of MHD waves has also been used to characterize the ion-neutral coupling. This ratio is known as the wave coupling number $W=R / \lambda_{0}$, where $\lambda_{0}=\frac{\pi V_{A}}{r_{m}}, V_{A}$ is the Alfven speed, and $v_{i n}$ is the ion-neutral collision frequency (Elmegreen \& Fiebig 1993; Myers \& Khersonsky 1995; Bergin et al. 1999). Since free MHD waves propagation in a clump requires minimum wave damping, we expect this minimum wavelength to be less than the observed length-scale, or $W>1$, as this ratio gives the range between the maximum possible wavelength (core size) and the cutoff wavelength (Kulsrud \& Pearce 1969; Elmegreen \& Fiebig 1993). Thus, when $W \gg 1$ we expect that the field and the gas are coupled over a large range of length-scales. Using our previous determination for the strength of the magnetic field in this clump, the Alfven speed, and the ion-neutral collision frequency we obtain a value for the wave coupling number of $W=110$. Previous determinations of $W$ suggest that values close 1 are obtained when the 
field is in equipartition with self-gravity; while greater values are consistent with a dominant magnetic field and strong coupling between the field and the neutral gas. Values around $W \sim 10^{3}$ have been found toward dense cores (Myers \& Khersonsky 1995), but smaller values have also been obtained $(W \sim 20)$ for massive cores (Bergin et al. 1999). Values smaller than $10^{3}$ suggest marginal coupling, where MHD waves can still propagate, but damping by the field is still possible. We have obtained a result larger than the equipartition value, but still small in comparison with other dense cores. As we have previously found, the mass-to-magnetic-flux ratio in this clump is super critical and the dispersion function in the field lines, derived by PCI from the polarization map of Cortes \& Crutcher (2006), suggests that a significant fraction of the field is turbulent. Thus, a sligthly larger than 1 value for the ambipolar diffusion Reynolds number and a larger than 1 wave coupling number value suggest that in MM1 the neutral gas has already or is in the process of decoupling from the field and a transition between a more dominant field and the full onset of gravitational collapse is ongoing. However, there is still sufficient dynamic range in the length-scales for the gas and the field to move as a single system. It is interesting that we are seeing an infalling clump with a field just decoupling from the gas and a hot core already formed where molecular abundances are being changed by the central source(s) and where no powerful outflows have been confirmed yet. This points to an intermediate scenario for the evolution of a high mass star forming region. The clump seems to have evolved creating the inner source(s) before the envelope stops accreting and before the gas gets fully decoupled from the ambient magnetic field. Moreover and even though MM1 is collapsing, we see evidence for an envelope in which the ions have a more extended distribution than the neutrals from species excited at similar critical densities. In principle ambipolar difussion can distribute the magnetic flux to the envelope restricting the movement of ions while the neutrals collapse. This mechanism can operate in the presence of MHD turbulence in which numerical simulations suggests that the ambipolar diffusion time scale is significantly reduced, but it can also efficiently redistribute magnetic flux (Li et al. 2008). Nevertheless, we cannot rule out that the spatial differentiation seen here is produced by 
chemistry alone, we favor the previous mechanism as the more important one where chemical differentiation is primarily responsible for the spatial differentiation seen in MM1.

\section{Summary and Conclusions}

We have presented new and re-processed sub-millimeter data from W43-MM1. New $\mathrm{N}_{2} \mathrm{H}^{+}(4 \rightarrow 3), \mathrm{DCO}^{+}(5 \rightarrow 4)$, and $\mathrm{SiO}(8 \rightarrow 7)$ data have been obtained as well as previous un-published $\mathrm{C}^{18} \mathrm{O}(3 \rightarrow 2)$ observations. We have re-processed previous $\mathrm{HCO}^{+}(4 \rightarrow 3)$, $\mathrm{H}^{13} \mathrm{CO}^{+}(4 \rightarrow 3), \mathrm{HCN}(4 \rightarrow 3), \mathrm{H}^{13} \mathrm{CN}(4 \rightarrow 3)$, and $\mathrm{CS}(7 \rightarrow 6)$ data to obtain chemical abundances in MM1. New un-published results with the SMA, place a warm hot core, of about $400 \mathrm{~K}$, inside MM1 as well as an outflow (Sridharan to be submitted 2011). We did not detect $\mathrm{SiO}$ emission over the $3 \sigma$ threshold; thus, the outflow emission seen in the SMA data is not seen from single dish measurements as it is likely deep inside the clump or its emission is highly diluted within the single dish beam. We have calculated column densities by using the RADEX code, which agree with classical methods within a factor of 2. Abundances for these lines have been derived for 3 selected points along the main axis of the MM1 clump. We have compared our findings with chemical models by Doty et al. (2002); Rodgers \& Charnley (2003); Nomura \& Millar (2004); Roueff et al. (2007). Comparison with these models put the MM1 clump hot core within an evolutionary age of about $10^{4} \mathrm{yr}$. We also found the $\mathrm{N}_{2} \mathrm{H}^{+}(4 \rightarrow 3)$ peak emission offset from the dust peak by about $0.5 \mathrm{pc}$, which can be explained by $\mathrm{CO}$ enhancement at the MM1 center due to the hot core as $\mathrm{CO}$ quickly destroys $\mathrm{N}_{2} \mathrm{H}^{+}$. We derived a deuterium fractionation of 0.0012 based on our $\left[\mathrm{DCO}^{+}\right] /\left[\mathrm{HCO}^{+}\right]$abundance ratio. This fractionation is consistent with an already evolved clump in which the hot core is releasing $\mathrm{C}$ and $\mathrm{O}$ bearing molecules altering the chemical composition of the MM1 clump and is also consistent with the depletion of $\mathrm{N}_{2} \mathrm{H}^{+}$seen at the dust peak. These findings question how well the dust temperature is coupled to the gas temperature, as comparison with models suggest higher gas temperatures 
than the $19 \mathrm{~K}$ derived from the dust SED. We conclude that the gas should be warmer than the dust inside the clump as the dust emission might be likely optically thick. At the same time, our maps of integrated emission suggest different spatial distributions for ions and neutral species; while the ions are more wide-spread around the MM1 center, the neutrals (except $\mathrm{C}^{18} \mathrm{O}$ ) show a compact ditribution around the dust peak. It is possible that magnetic tension is restricting the ion movement while the neutrals collapse more freely, but we favor chemical differentation induced by the hot core as the main mechanism behind the spatial differentation seen in MM1. The $\mathrm{C}^{18} \mathrm{O}$ map suggests that the MM1 clump does not follow completely the filament suggested by the dust and the ${ }^{12} \mathrm{CO}$ maps. Two regions are seen, one corresponds to the MM1 clump and the other to what we believe is the interface with the H II region (a PDR?). If this is confirmed, then the shock front from the $\mathrm{H}$ II region has not yet reached the MM1 clump, which may have implications for triggered star formation models.

By using our derived abundances for the ions, we have derived an electron fraction of $X(e)=6.5 \times 10^{-8}$ where we used an average value for the $\mathrm{H}_{2} \mathrm{D}^{+}$abundance from Caselli et al. (2008) and our deuterium fraction to derive the $\mathrm{H}_{3}^{+}$abundance. Our value for $X(e)$ is consistent with similar values in other high mass star forming regions found in the literature. Using the PCI estimation for the magnetic field strength, we calculated an ambipolar diffusion Reynolds number $R_{m}=18.1$, a Hartmann number $M=7 \times 10^{6}$, and a wave coupling number $W=110$. These values suggest that the field has or is in the process of decoupling from the fluid as infall proceeds. As the Reynolds number is still not comparable with the Hartmann number, the field may still damp MHD waves, but as suggested by the wave coupling number, there is still enough dynamic range in the length-scales for the field and the fluid to move as a single system. By combining our findings here, we believe that the MM1 clump is in an intermediate stage of evolution. At an intermediate stage of evolution, the inner source(s) has(have) formed a hot core where a strong outflow is detected. The envelope is still contracting and it is not fully decoupled from the ambient magnetic field. To explore higher resolution spatial scales, particularly at the cutoff 
(minimum wavelength) level, interferometric observations will be needed. Thus, ALMA is the perfect instrument to study W43 further.

The National Radio Astronomy Observatory is a facility of the National Science Foundation operated under cooperative agreement by Associated Universities, Inc. 


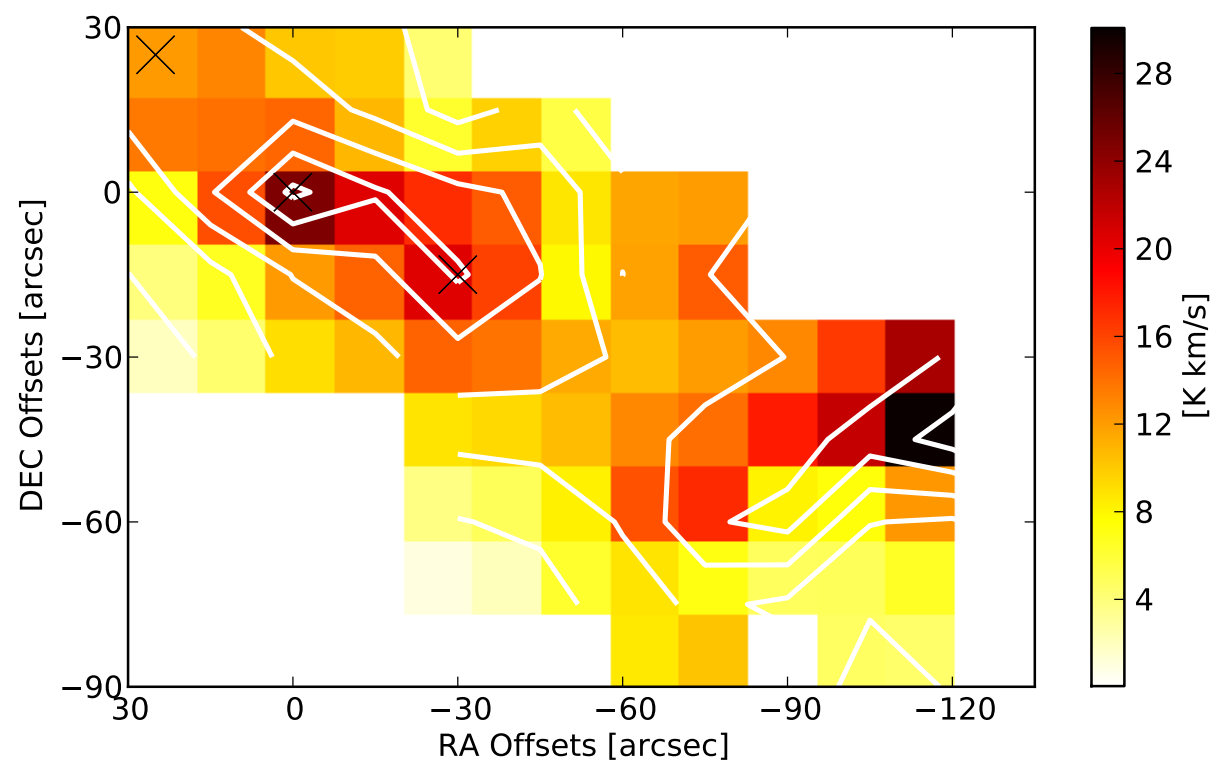

Fig. 1.- A $60^{\prime \prime} \times 60^{\prime \prime}$ integrated intensity map of $\mathrm{C}^{18} \mathrm{O}(3 \rightarrow 2)$ from W43-MM1 centered at the dust peak. The color scale correspond to the integrated emission after applying a nearest interpolation scheme. The contours are choosen to be $8,12,16,20$, and $24 \mathrm{~K} \mathrm{~km} \mathrm{~s}^{-1}$ while the colorscale is indicated in the colorbar. Crosses indicate the positions at which we derived fractional abundances from our sample of molecules. The offsets are $\left(30^{\prime \prime}, 30^{\prime \prime}\right),\left(0^{\prime \prime}, 0^{\prime \prime}\right)$, and $\left(-30^{\prime \prime}, 15^{\prime \prime}\right)$ in $(\alpha, \delta)$. Note tha the reference position is offset from the center of the map by $60^{\prime \prime}$ in $\alpha$ and $30^{\prime \prime}$ in $\delta$. 


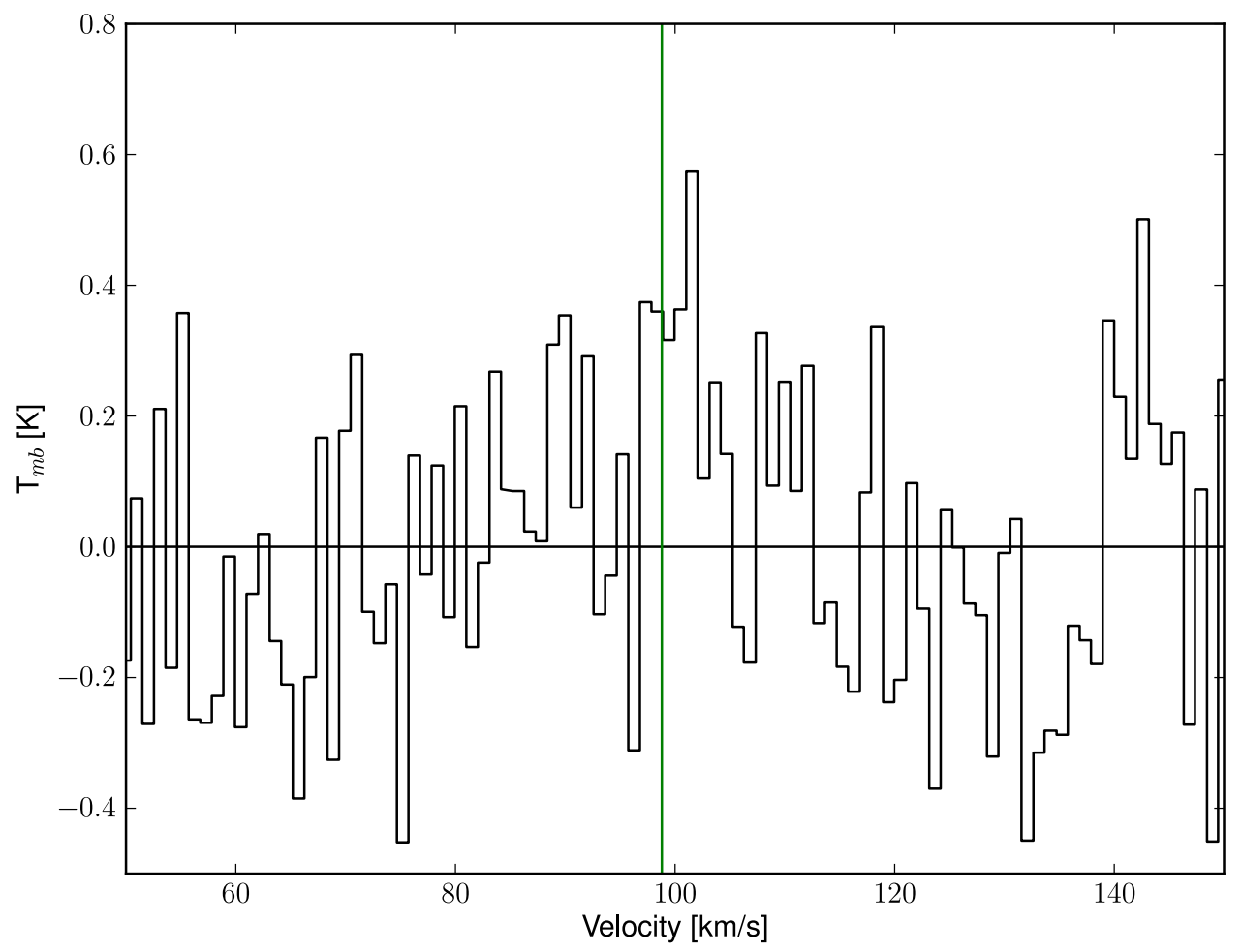

Fig. 2.- The figure shows our $\mathrm{SiO}(8 \rightarrow 7)$ spectrum towards W43-MM1. The black line marks the zero baseline and the systemic velocity of the clump, $98.9 \mathrm{~km} \mathrm{~s}^{-1}$ is shown by the green line. The spectra is binned every 10 channels giving a resolution in velocity of about $1 \mathrm{~km} \mathrm{~s}^{-1}$ giving and rms noise of $0.24 \mathrm{~K}$. The intensity upper limit at the systemic velocity is $0.6 \mathrm{~K}$. 


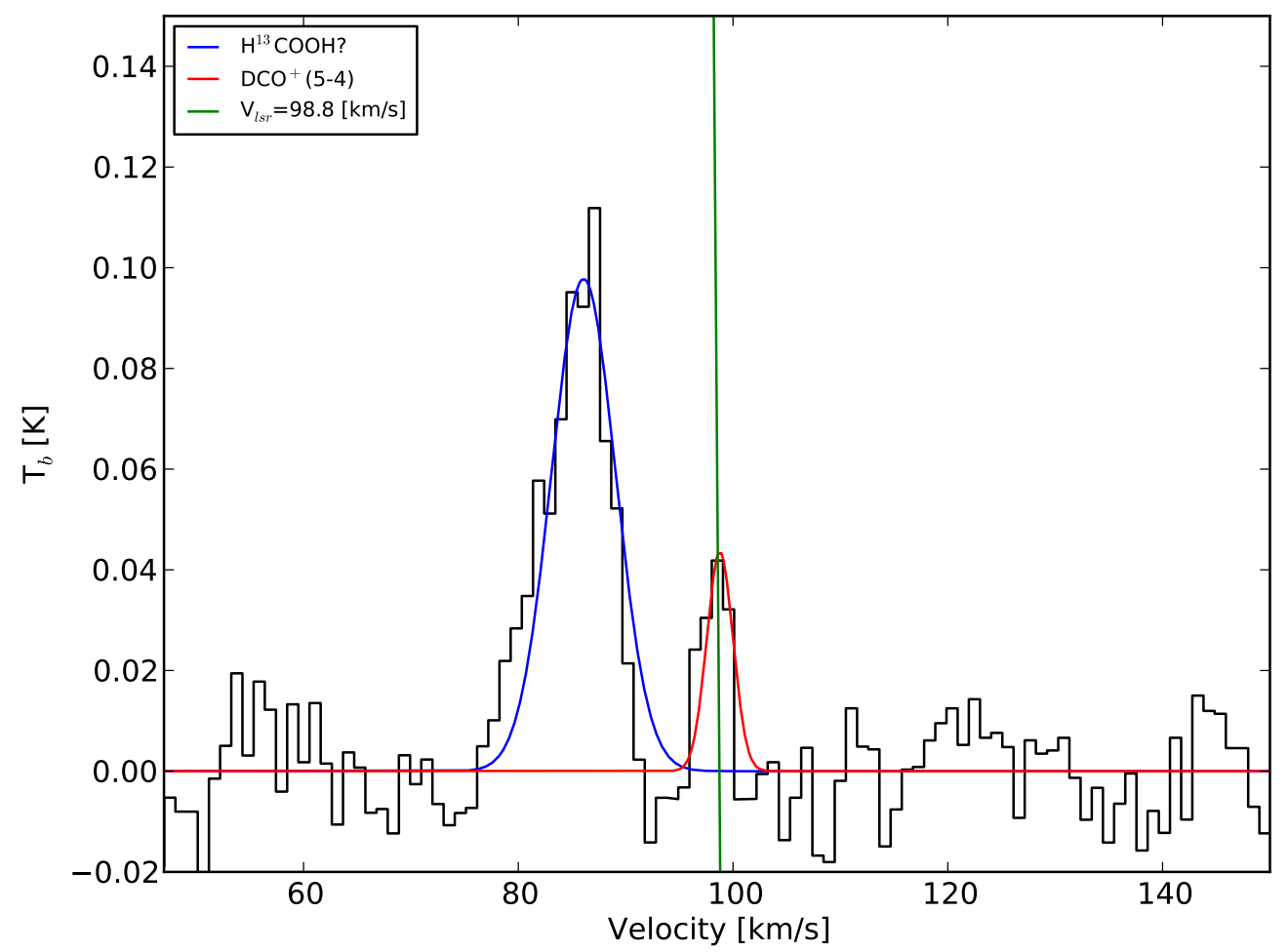

Fig. 3.- The spectrum shows our $\mathrm{DCO}^{+}(5 \rightarrow 4)$ detection towards W43-MM1. The Gaussian fit is shown by the red curve, note that the systemic velocity of the clump, $98.9 \mathrm{~km} \mathrm{~s}^{-1}$ is shown by the green line. Also, the un-identified line is also shown with its corresponding Gaussian fit (blue curve). 


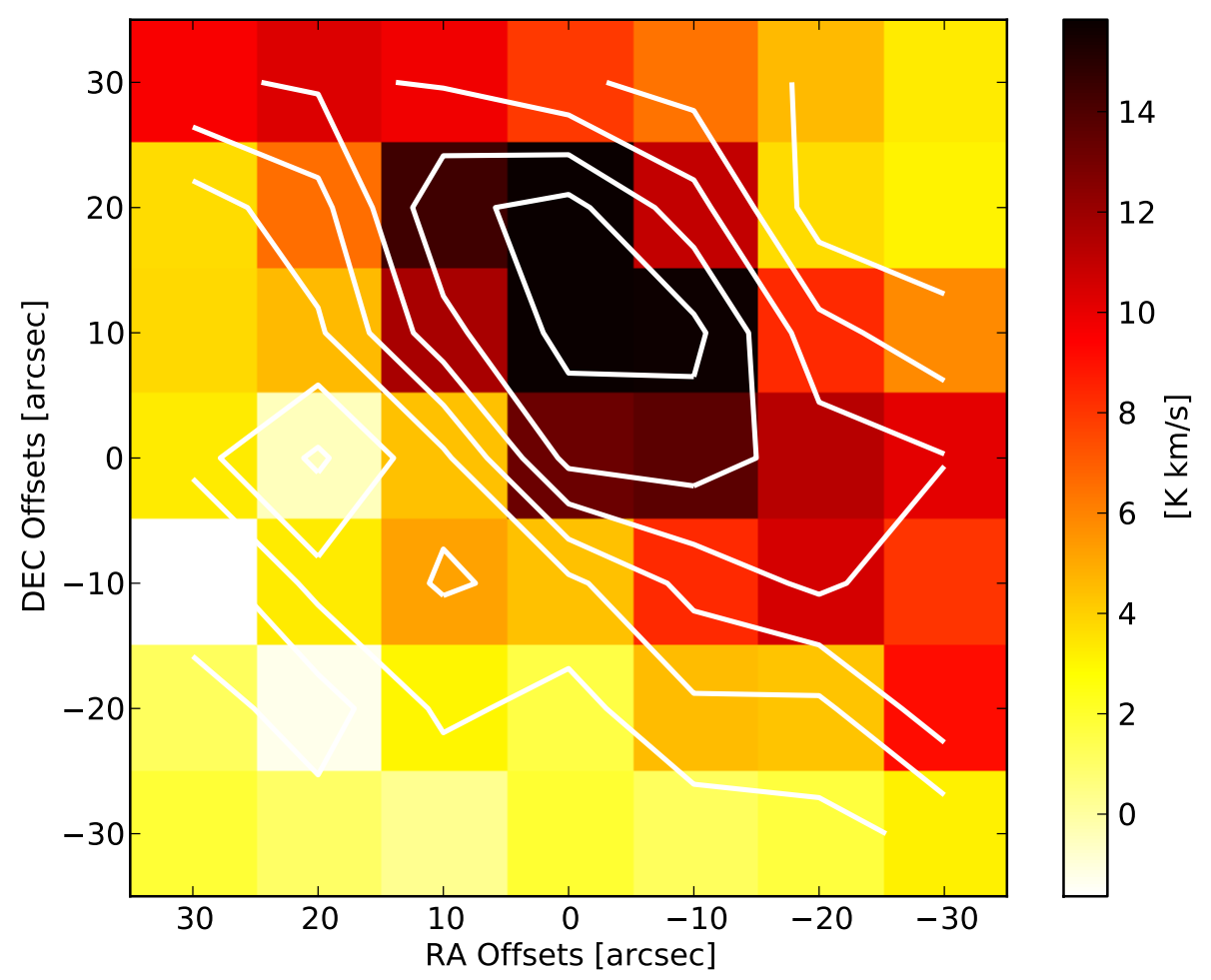

Fig. 4.- A $60^{\prime \prime} \times 60^{\prime \prime}$ integrated intensity map of $\mathrm{N}_{2} \mathrm{H}^{+}(4 \rightarrow 3)$ from W43-MM1 centered at the dust peak is shown. The color scale correspond to the integrated emission after applying a nearest interpolation scheme in units of $\mathrm{K} \mathrm{km} \mathrm{s}^{-1}$. The contours are chosen to be 2.5, 5, 7.5, 10, 12.5, and $15 \mathrm{~K} \mathrm{~km} \mathrm{~s}^{-1}$. 


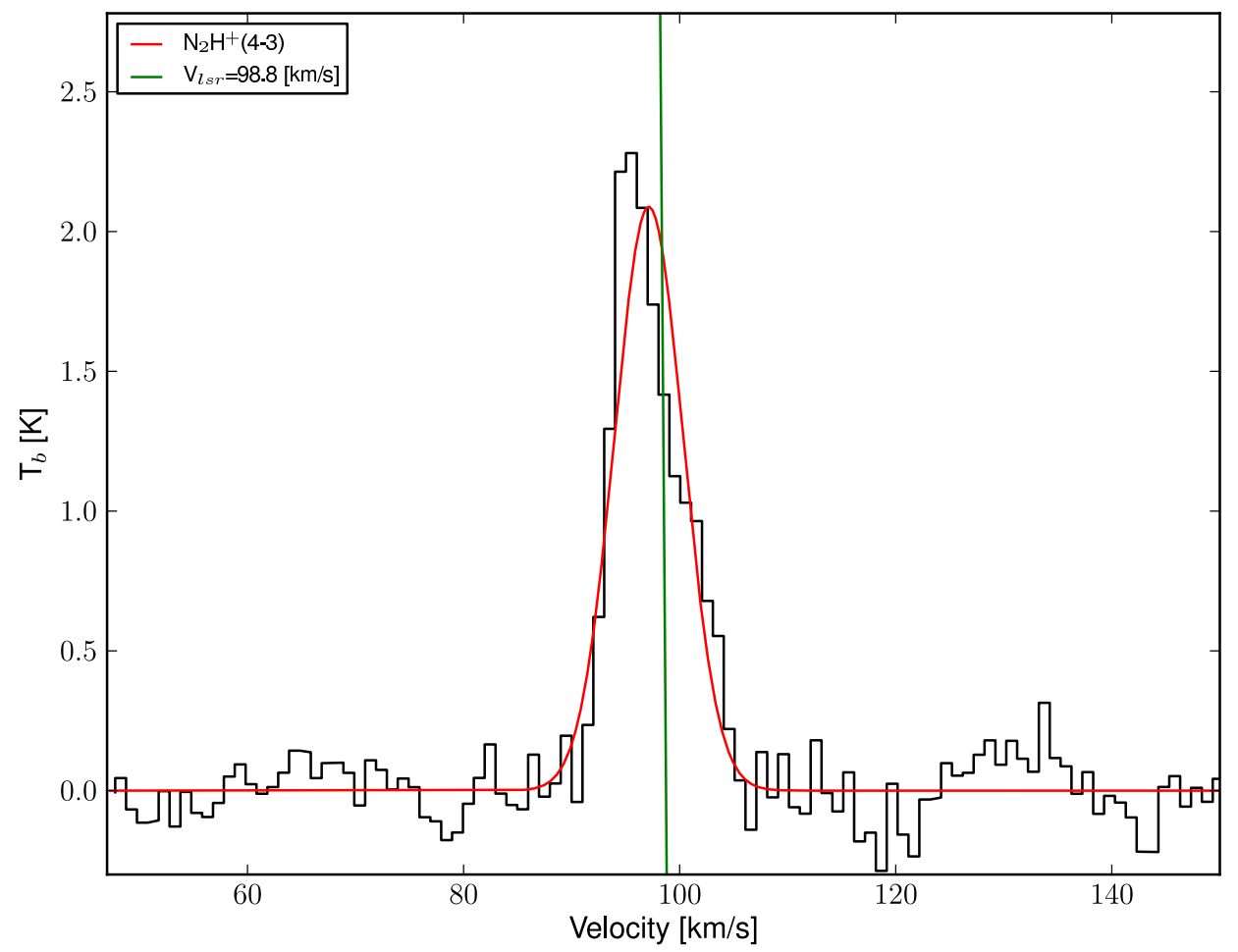

Fig. 5.- The spectrum shows the $\mathrm{N}_{2} \mathrm{H}^{+}(4 \rightarrow 3)$ from the center of W43-MM1. The Gaussian fit is shown by the red curve, note that the systemic velocity of the clump, $98.9 \mathrm{~km} \mathrm{~s}^{-1}$ is shown by the green line. The spectra is binned every 10 channels giving a resolution in velocity of about 1 $\mathrm{km} \mathrm{s}^{-1}$. The spectrum was obtained with a higher signal to noise than the data presented in Figure 4 as we integrated additional time at the center of MM1. 

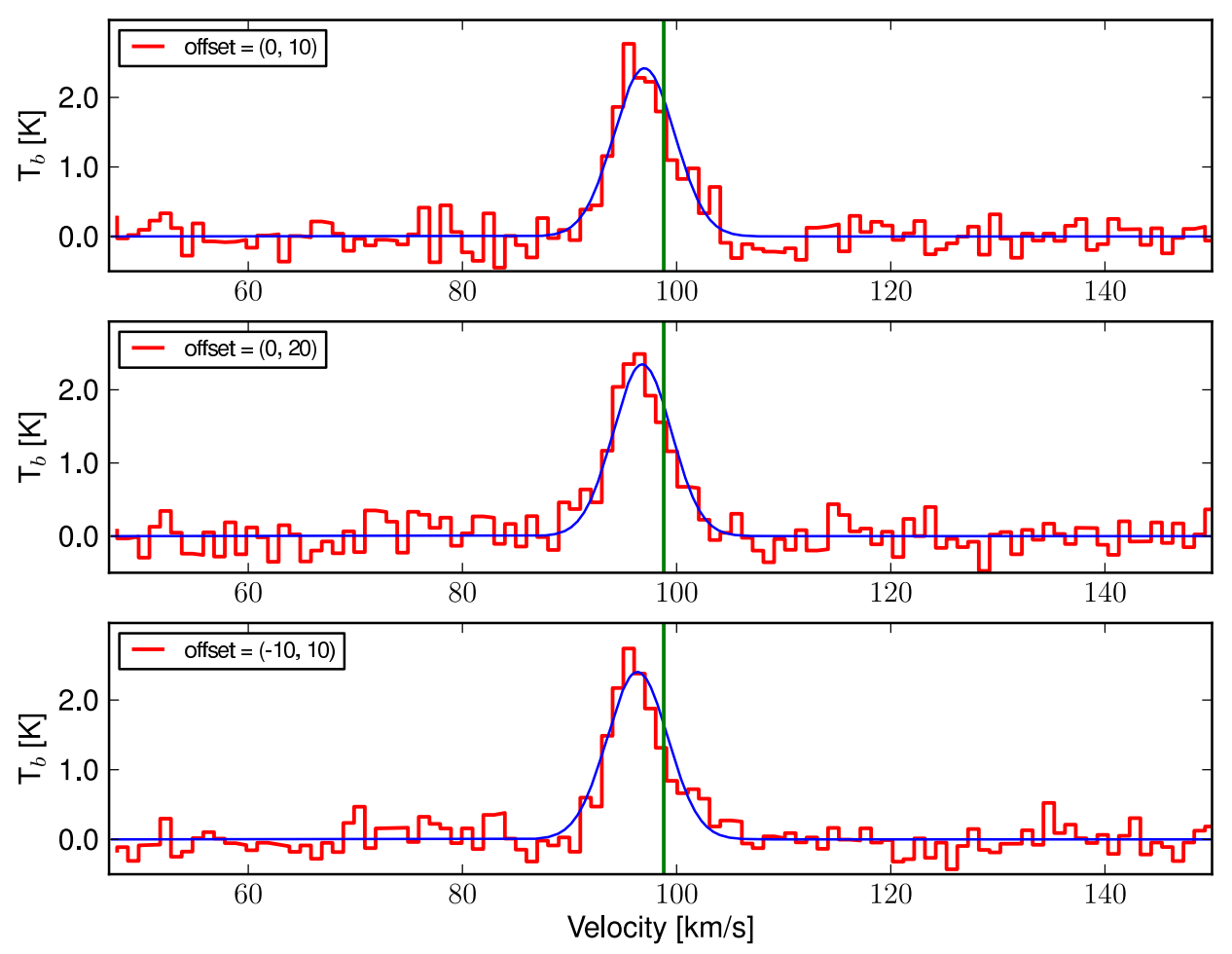

Fig. 6.- Spectra from $\mathrm{N}_{2} \mathrm{H}^{+}(4 \rightarrow 3)$ towards selected pointings in W43-MM1. The offsets are respect to the main reference position an indicated by the label box in each spectrum. The spectra is binned every 10 channels giving a resolution in velocity of about $1 \mathrm{~km} \mathrm{~s}^{-1}$. 


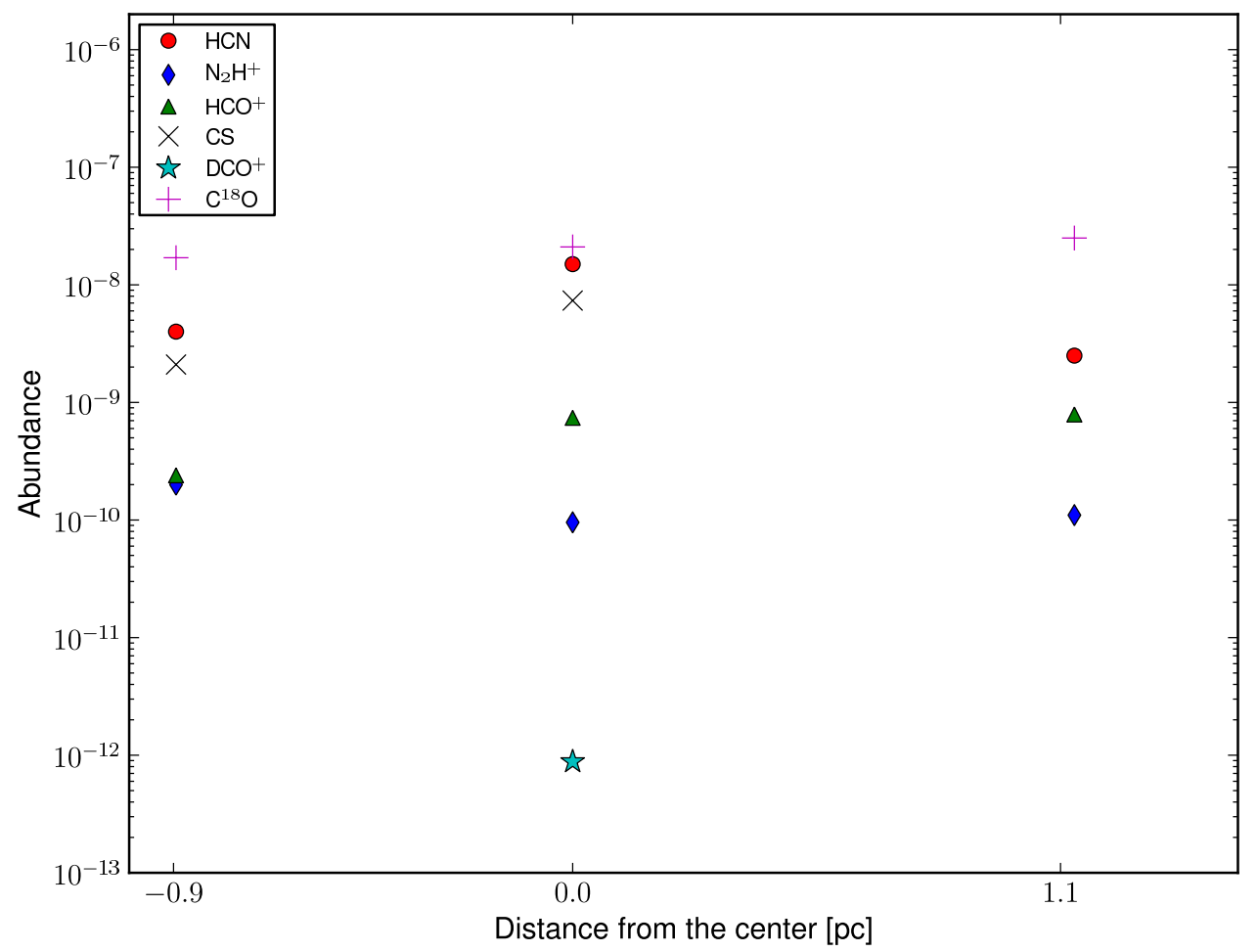

Fig. 7.- The plot shows the fractional abundances derived for all the species observed at the three selected pointings along the MM1 strip (main axis). The points are indicated by their relative distance respect to the center. Thus, $\left(30^{\prime \prime}, 30^{\prime \prime}\right)$ is at $1.1 \mathrm{pc}$, while $(-30,-15)$ is at $-0.9 \mathrm{pc}$ from the center. Each molecule is represented by a symbol indicated in upper left box. 


\section{REFERENCES}

Arce, H. G., Santiago-García, J., Jørgensen, J. K., Tafalla, M., \& Bachiller, R. 2008, ApJ, 681, L21

Bergin, E. A., Plume, R., Williams, J. P., \& Myers, P. C. 1999, ApJ, 512, 724

Bergin, E. A. \& Tafalla, M. 2007, ARA\&A, 45, 339

Bergman, P., Parise, B., Liseau, R., \& Larsson, B. 2011, A\&A, 527, A39+

Busquet, G., Estalella, R., Zhang, Q., Viti, S., Palau, A., Ho, P. T. P., \& Sánchez-Monge, Á. 2011, $\mathrm{A} \& \mathrm{~A}, 525, \mathrm{~A} 141+$

Caselli, P., Vastel, C., Ceccarelli, C., van der Tak, F. F. S., Crapsi, A., \& Bacmann, A. 2008, A\&A, 492, 703

Caselli, P., Walmsley, C. M., Zucconi, A., Tafalla, M., Dore, L., \& Myers, P. C. 2002a, ApJ, 565, 331

-. 2002b, ApJ, 565, 344

Cesaroni, R., Palagi, F., Felli, M., Catarzi, M., Comoretto, G., di Francos, Giovanardi, C., \& Palla, F. 1988, A\&AS, 76, 445

Chen, H., Liu, S., Su, Y., \& Zhang, Q. 2010, ApJ, 713, L50

Cortes, P., Crutcher, R. M. Shepherd, D., \& Bronfman, L. 2008, ApJ, 650, 1

Cortes, P. \& Crutcher, R. M. 2006, ApJ, 639, 965

Cortes, P. C., Crutcher, R. M., \& Watson, W. D. 2005, ApJ, 628, 780

Cortes, P. C., Parra, R., Cortes, J. R., \& Hardy, E. 2010, ArXiv e-prints 
Crapsi, A., Caselli, P., Walmsley, C. M., Tafalla, M., Lee, C. W., Bourke, T. L., \& Myers, P. C. 2004, A\&A, 420, 957

Crutcher, R. M., Troland, T. H., Lazareff, B., \& Kazes, I. 1996, ApJ, 456, 217

Crutcher, R. M., Troland, T. H., Lazareff, B., Paubert, G., \& Kazès, I. 1999, ApJ, 514, L121

Daniel, F., Cernicharo, J., Roueff, E., Gerin, M., \& Dubernet, M. L. 2007, ApJ, 667, 980

Davidson, P. A. 2001, An Introduction to Magnetohydrodynamics, ed. Davidson, P. A.

Doty, S. D., van Dishoeck, E. F., van der Tak, F. F. S., \& Boonman, A. M. S. 2002, A\&A, 389, 446

Elmegreen, B. G. \& Fiebig, D. 1993, A\&A, 270, 397

Fontani, F., Caselli, P., Crapsi, A., Cesaroni, R., Molinari, S., Testi, L., \& Brand, J. 2006, A\&A, 460,709

Friesen, R. K., Di Francesco, J., Myers, P. C., Belloche, A., Shirley, Y. L., Bourke, T. L., \& André, P. 2010, ApJ, 718, 666

Fuller, G. A., Williams, S. J., \& Sridharan, T. K. 2005, A\&A, 442, 949

Garrod, R. T., Weaver, S. L. W., \& Herbst, E. 2008, ApJ, 682, 283

Güsten, R., Nyman, L. Å., Schilke, P., Menten, K., Cesarsky, C., \& Booth, R. 2006, A\&A, 454, L13

Herbst, E. \& van Dishoeck, E. F. 2009, ARA\&A, 47, 427

Hezareh, T., Houde, M., McCoey, C., Vastel, C., \& Peng, R. 2008, ApJ, 684, 1221

Hoare, M. G., Kurtz, S. E., Lizano, S., Keto, E., \& Hofner, P. 2007, in Protostars and Planets V, ed. B. Reipurth, D. Jewitt, \& K. Keil, 181-196 
Houde, M., Bastien, P., Peng, R., Phillips, T. G., \& Yoshida, H. 2000a, ApJ, 536, 857

Houde, M., Peng, R., Phillips, T. G., Bastien, P., \& Yoshida, H. 2000b, ApJ, 537, 245

Johnstone, D., Rosolowsky, E., Tafalla, M., \& Kirk, H. 2010, ApJ, 711, 655

Jørgensen, J. K., Schöier, F. L., \& van Dishoeck, E. F. 2004, A\&A, 416, 603

Kohno, K. 2005, in Astronomical Society of the Pacific Conference Series, Vol. 344, The Cool Universe: Observing Cosmic Dawn, ed. C. Lidman \& D. Alloin, 242-+

Kulsrud, R. \& Pearce, W. P. 1969, ApJ, 156, 445

Lai, S. P. 2001, PhD thesis, University of Illinois at Urbana - Champaign, Urbana, IL 61801, available at the Astronomy library at the Astronomy building

Li, H.-b., Houde, M., Lai, S.-p., \& Sridharan, T. K. 2010, ApJ, 718, 905

Li, P. S., McKee, C. F., Klein, R. I., \& Fisher, R. T. 2008, ApJ, 684, 380

Lintott, C. J., Viti, S., Rawlings, J. M. C., Williams, D. A., Hartquist, T. W., Caselli, P., Zinchenko, I., \& Myers, P. 2005, ApJ, 620, 795

Liszt, H. S. 1995, AJ, 109, 1204

Liu, S., Girart, J. M., Remijan, A., \& Snyder, L. E. 2002, ApJ, 576, 255

McDaniel, E. W. \& Mason, E. A. 1973, The Mobility and Diffusion of Ions in Gases (New York Wiley-Interscience, 1973.)

McKee, C. F., Li, P. S., \& Klein, R. I. 2010, ApJ, 720, 1612

McKee, C. F. \& Ostriker, E. C. 2007, ARA\&A, 45, 565

Mezger, P. G., Zylka, R., \& Wink, J. E. 1990, A\&A, 228, 95 
Miettinen, O., Harju, J., Haikala, L. K., Kainulainen, J., \& Johansson, L. E. B. 2009, A\&A, 500, 845

Mooney, T., Sievers, A., Mezger, P. G., Solomon, P. M., Kreysa, E., Haslam, C. G. T., \& Lemke, R. 1995, A\&A, 299, 869

Motte, F., Schilke, P., \& Lis, D. C. 2003, ApJ, 582, 277

Mouschovias, T. C. \& Paleologou, E. V. 1981, ApJ, 246, 48

Muller, H. S., Schlder, F., Stutzki, J., \& Winnewisser, G. 2005, Journal of Molecular Structure, 742, 215 , mOLECULAR SPECTROSCOPY AND STRUCTURE - A Collection of Invited Papers in Honor of Dr. Walter J. Lafferty

Myers, P. C. \& Khersonsky, V. K. 1995, ApJ, 442, 186

Nomura, H. \& Millar, T. J. 2004, A\&A, 414, 409

Pirogov, L., Zinchenko, I., Caselli, P., \& Johansson, L. E. B. 2007, A\&A, 461, 523

Remijan, A. J., Markwick-Kemper, A., \& ALMA Working Group on Spectral Line Frequencies. 2007, in Bulletin of the American Astronomical Society, Vol. 38, Bulletin of the American Astronomical Society, 963-+

Roberts, H. \& Millar, T. J. 2000, A\&A, 361, 388

Rodgers, S. D. \& Charnley, S. B. 2001, ApJ, 546, 324

-. 2003, ApJ, 585, 355

Roueff, E., Parise, B., \& Herbst, E. 2007, A\&A, 464, 245

Sakai, T., Sakai, N., Hirota, T., \& Yamamoto, S. 2010, ApJ, 714, 1658

Schöier, F. L., van der Tak, F. F. S., van Dishoeck, E. F., \& Black, J. H. 2005, A\&A, 432, 369 
Smith, L. F., Biermann, P., \& Mezger, P. G. 1978, A\&A, 66, 65

Sridharan to be submitted, T. K. 2011, ApJ, 442, 186

Szymczak, M., Bartkiewicz, A., \& Richards, A. M. S. 2007, A\&A, 468, 617

Turner, B. E. 2001, ApJS, 136, 579

Wakelam, V., Caselli, P., Ceccarelli, C., Herbst, E., \& Castets, A. 2004, A\&A, 422, 159

Williams, J. P., Bergin, E. A., Caselli, P., Myers, P. C., \& Plume, R. 1998, ApJ, 503, 689

Wilson, T. L., Mezger, P. G., Gardner, F. F., \& Milne, D. K. 1970, A\&A, 6, 364

Wilson, T. L. \& Rood, R. 1994, ARA\&A, 32, 191

Wood, D. O. S. \& Churchwell, E. 1989, ApJS, 69, 831

Wootten, A., Snell, R., \& Glassgold, A. E. 1979, ApJ, 234, 876

Zinchenko, I., Caselli, P., \& Pirogov, L. 2009, MNRAS, 395, 2234

Zweibel, E. G. 2002, ApJ, 567, 962 

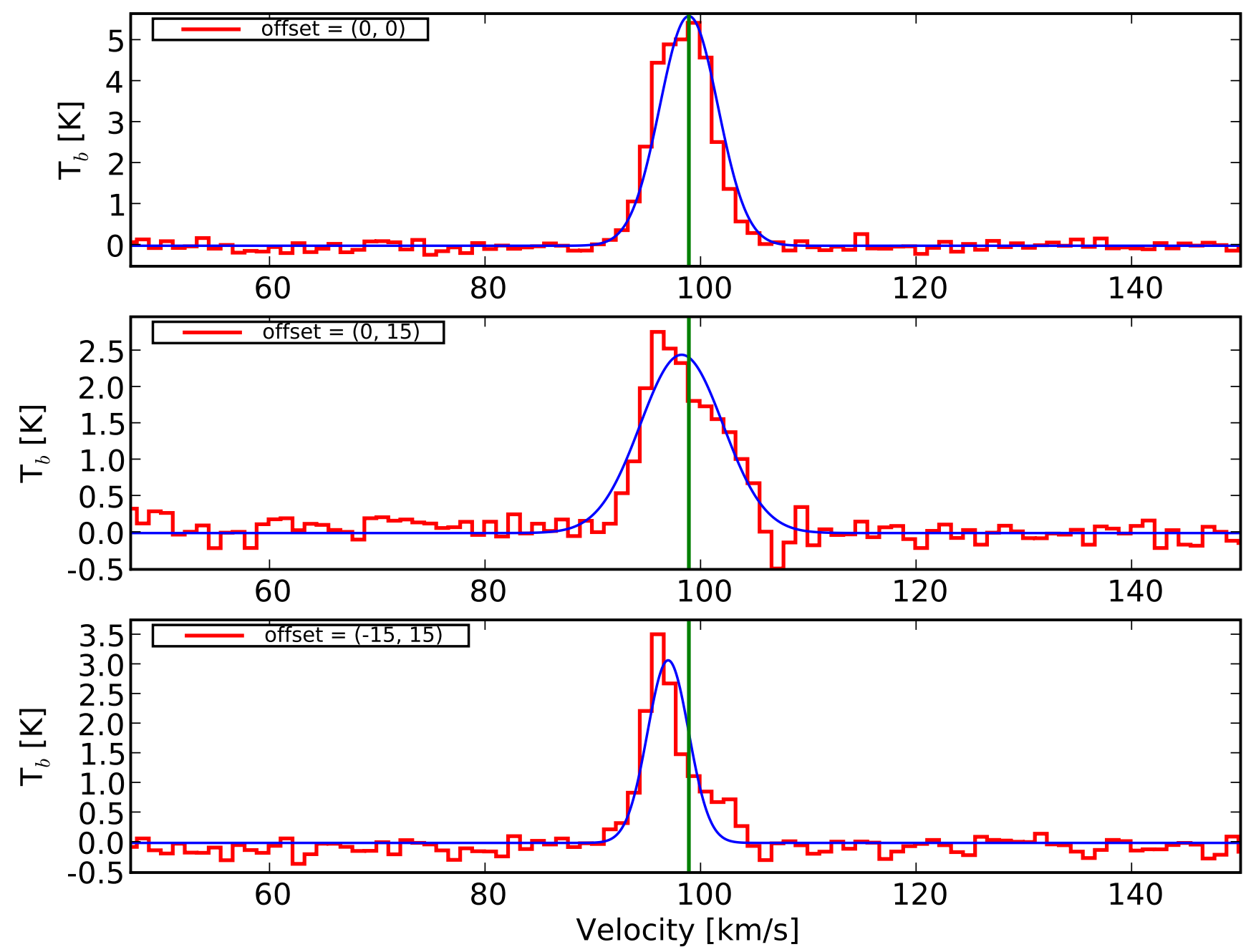


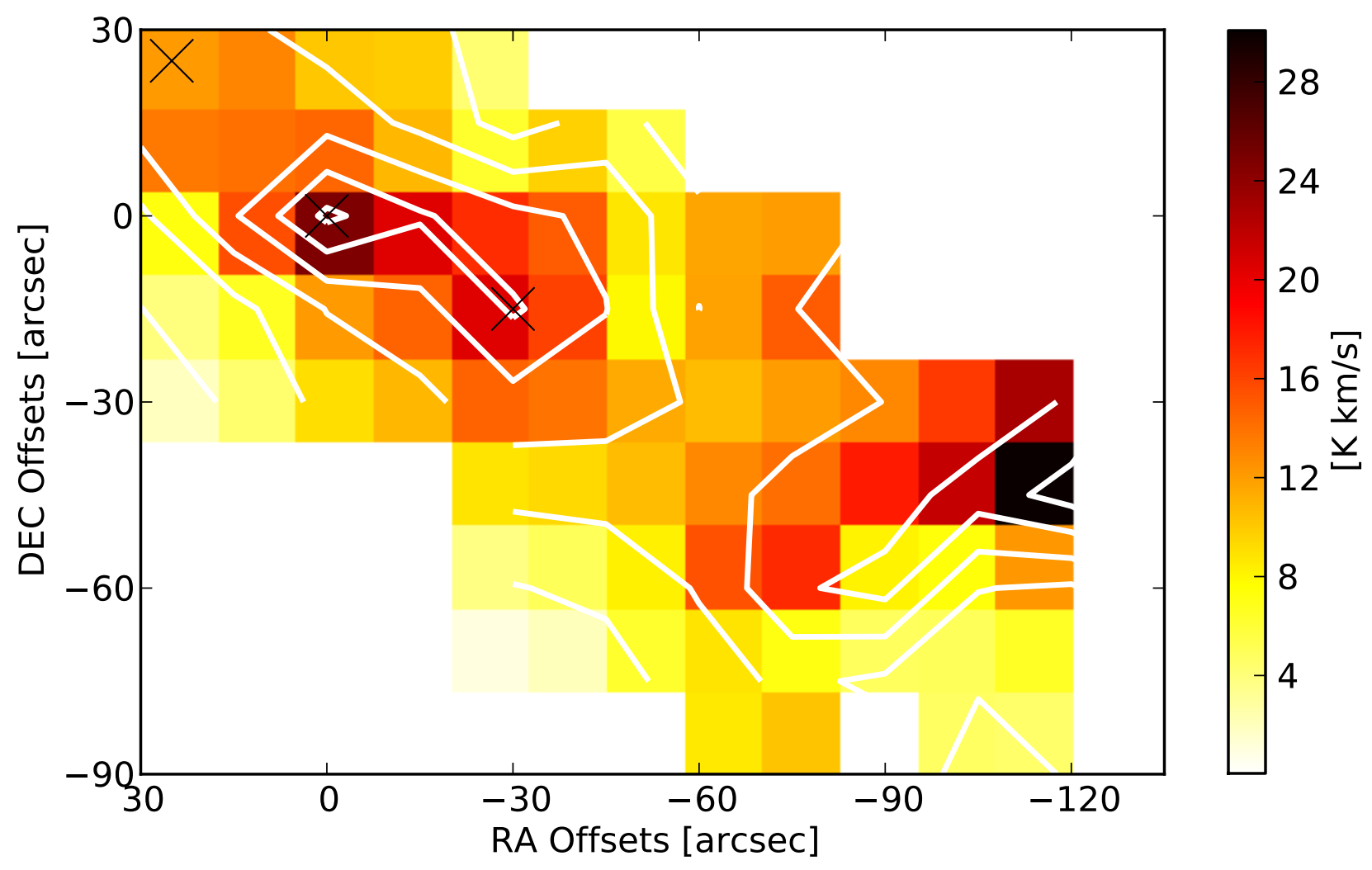

